\title{
Self-interacting scalar fields at high-temperature
}

\author{
Alexandre Deur (i) $^{\mathrm{a}}$ \\ University of Virginia, Charlottesville, VA 22904, USA
}

Received: 2 March 2017 / Accepted: 4 June 2017 / Published online: 19 June 2017

(C) The Author(s) 2017. This article is an open access publication

\begin{abstract}
We study two self-interacting scalar field theories in their high-temperature limit using path integrals on a lattice. We first discuss the formalism and recover known potentials to validate the method. We then discuss how these theories can model, in the high-temperature limit, the strong interaction and General Relativity. For the strong interaction, the model recovers the known phenomenology of the nearly static regime of heavy quarkonia. The model also exposes a possible origin for the emergence of the confinement scale from the approximately conformal Lagrangian. Aside from such possible insights, the main purpose of addressing the strong interaction here - given that more sophisticated approaches already exist - is mostly to further verify the pertinence of the model in the more complex case of General Relativity for which non-perturbative methods are not as developed. The results have important implications on the nature of Dark Matter. In particular, non-perturbative effects naturally provide flat rotation curves for disk galaxies, without need for non-baryonic matter, and explain as well other observations involving Dark Matter such as cluster dynamics or the dark mass of elliptical galaxies.
\end{abstract}

\section{Introduction}

The nature of confinement in a Yang-Mills theory is an important and complex question that is not settled yet. In fact, there is no consensus on what mechanism leads to confinement. Various candidates include center vortices, magnetic monopoles, Abelian Higgs mechanism, the Gribov mechanism, or the creation of an Abrikosov-type string via dual Meissner effect; see [1] for a review. Even the phenomenology of the strong interaction's strings/flux tubes is challenged as a manifestation of quark confinement [2]. What is clear is that confinement stems from field self-interactions in the non-perturbative, i.e., the strong coupling, regime.

\footnotetext{
a e-mail: deurpam@jlab.org
}

In this paper, we numerically study two self-interacting scalar field theories which may correspond, in the hightemperature limit, to Quantum Chromodynamics (QCD) and General Relativity (GR). QCD is the non-Abelian - and thus self-interacting - theory of the strong interaction and the prototypical confining theory. Its phenomenology has been - and continue to be - experimentally scrutinized. Many methods have been developed to study it. They have reached a high degree of sophistication. Thus, our primary purpose here is not to study QCD with a simplified approach but rather to use the strong interaction as a testing ground, once our model is checked with simpler free-field theories. Nevertheless, we hope that the present simple model can yield interesting insight in the confinement mechanism. Gravity's GR is also a self-interacting theory which ensuing non-linearities make its resolution notoriously difficult. Studying it is not as developed as for QCD and our model provides a relatively simple way to approach its phenomenology. The verification of our approach in the free-field case and its relevance to QCD phenomenology, indicate that its predictions for GR should also be pertinent.

The paper is organized as follows. We first introduce the Lagrangians of two scalar self-interacting field theories. Next, the corresponding instantaneous potentials are expressed in terms of path integrals on a lattice, and calculated with a standard numerical Monte-Carlo technique. We then interpret the results obtained in the high-temperature regime. Finally, we discuss how the scalar theories may approximate the non-zero spin field theories of QCD (spin 1, i.e. vector-field) and GR (spin 2, i.e. rank-2 tensor-field). In particular, we recall how the Lagrangian of GR can be expressed in a polynomial form which, when expressed in the static limit, is identical to one of the scalar Lagrangians previously introduced in the high-temperature limit. A first consequence of the high-temperature limit is that numerical calculations become extremely fast and tractable. Furthermore, the high-temperature limit takes the innate quantum nature of a path-integral formalism to the classical limit. 
While this is a limitation for modeling the inherently quantum strong interaction, it is advantageous for approximating GR since it alleviates the notorious difficulties associated with quantum gravity. The strong interaction being intrinsically non-classical, there is no reason to expect that the high-temperature potential corresponds to the known quarkquark phenomenological static potential. It is in fact not the case: the high-temperature (i.e. classical) potential derived with the method discussed in this article has approximately a Yukawa form, which is very different from the phenomenological quark-quark linear potential. Although the classical potential cannot be compared to the phenomenological one, it is still a valuable result since it can be compared to results from other approaches obtained in the classical limit of QCD. An agreement would then validate the method used in this article. Furthermore, the short distance quantum effects can be introduced had-oc by allowing the theory's coupling to run. Checking whether such running transforms the Yukawa potential into the expected linear potential would then validate further the approach and makes it relevant to the study of confinement. As we already made clear, the method discussed here in the context of the strong interaction is not intended to compete with the more mature and sophisticated approaches that are already used to study the strong interaction, such as Lattice QCD, the Schwinger-Dyson approach or the Anti-de Sitter/Conformal Field Theory (AdS/CFT) duality. Rather, the primary purpose of studying the strong interaction is to check the technique so that it can be applied with confidence to the more complex - but classical - case of GR. A secondary benefit is that new approaches may provide fresh views on mechanisms important to the confinement problem. As a specific example, it is interesting to see in the approach discussed here how a mass scale emerges out of a conformal Lagrangian. Such a phenomenon is at the heart of recent advances in our understanding of QCD in its non-perturbative regime [3]. At the end of this article, after having validated our approach using the free-field and QCD cases, we discuss the predictions of our model in the GR case. In particular, the model naturally explains many cosmological observations without need for non-baryonic dark matter. To show how these observations can be naturally and accurately explained by a phenomenology well-known in the QCD context is the main goal of this article. These observations include the rotation curves of disk galaxies, the fact that they are approximately flat, The correlation between the rotation speeds and baryonic masses of disk galaxies [4,5], the correlation between the dark mass of elliptical galaxies and their ellipticities [6], the dynamics of galaxy clusters and the Bullet cluster observations [7].

We have grouped the technical aspects of this work in several appendices: In Appendix A we recall the numerical technique used to obtain the potentials. In Appendix B we give step-by-step instructions for programming a basic

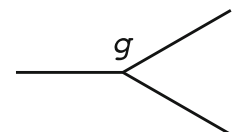

1) $\partial_{\mu} \phi \partial^{\mu} \phi$

2) $\partial_{\mu} \phi \partial^{\mu} \phi$

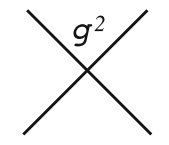

$\phi^{4}$

$\phi^{2} \partial_{\mu} \phi \partial^{\mu} \phi$
Fig. 1 Graphs used to construct the Lagrangians. The graph on the left corresponds to the free part of the theory yielding a $1 / r$ potential. The two other graphs create field self-interactions that distort this potential. In case (1), the forms of these terms are imposed by choosing a dimensionless coupling (no consistent cubic term can be formed). In case (2) they are chosen for a coupling of dimension [energy $]^{-2}$

calculation. In Appendix C, we discuss the question of the choice of boundary conditions and show the independence of our results on such choices. In Appendix D, we recover several analytically known potentials to validate the technique developed in this paper. In Appendix E, we verify that the results are independent of the choice of lattice parameters, such as mesh spacing, decorrelation coefficient or lattice size. In Appendix F we recover the post-Newtonian formalism from the scalar Lagrangian, thereby further verifying that it correctly approximates GR in the classical limit.

This approach is simple and fast. We give in Appendix B the time necessary for a standard computer to perform a calculation to good precision. Extending this figure to state-ofart lattice machines implies that a one minute calculation would reach a relative precision of $2 \times 10^{-7}$, comparable to the intrinsic precision of a 32-bit machine. It should be remarked that the method and algorithms are not specially optimized, since the speed is adequate for the problems discussed in this paper.

\section{Scalar Lagrangians}

Confinement stems from field self-interactions in large coupling or strong field regimes. Lagrangian densities for selfinteracting theories can be constructed from the graphs shown in Fig. 1. We discuss here the simple case of a scalar (spin 0) field $\phi$. We consider only massless fields, like those describing the QCD and GR forces.

The form of the Lagrangian density in case (1) is

$\mathcal{L}=\partial_{\mu} \phi \partial^{\mu} \phi-g^{2} \phi^{4}$,

with $g$ the coupling, dimensionless as for QCD. For case (2) the Lagrangian density is

$\mathcal{L}=\partial_{\mu} \phi \partial^{\mu} \phi+g \phi \partial_{\mu} \phi \partial^{\mu} \phi+g^{2} \phi^{2} \partial_{\mu} \phi \partial^{\mu} \phi$,

with $g$ a coupling of dimension [energy] ${ }^{-2}$, as for GR.

There is no cubic term in Eq. (1) since it would not be Lorentz invariant. One could insist on having such a term by adding a unit 4-vector $e^{\mu}$ to make the Lorentz structure of 
Eq. (1) internally consistent when a cubic term is included. However, such a $g \phi^{2} \partial_{\mu} \phi e^{\mu}$ term would have no influence since it disappears from the Euler-Lagrange equation:

$$
\frac{\partial \mathcal{L}}{\partial \phi}-\partial_{\mu} \frac{\partial \mathcal{L}}{\partial \partial^{\mu} \phi}=-2 g^{2} \phi^{3}-\partial^{2} \phi,
$$

as we see from the absence of a term linear in $g$. Only the terms stemming from the quartic component (proportional to $g^{2}$ ) and from the quadratic one remain.

Although the scalar field Lagrangians in Eqs. (1) and (2) stem from the same diagrams, they display different structures. There is no cubic term in Eq. (1) and the quartic term contains no partial derivatives. Hence, it does not contribute to the propagation of the field and acts similarly to a mass term. In fact, the analytical solution for the equation of motion is known, with the field obeying a massive dispersion relation [8], whose resulting potential can be well fit by a Yukawa potential, as we show in Sect. 4.1. In Eq. (2), the cubic term is preserved. All terms contain partial derivatives and thus contribute to the field propagation.

\section{Computation of the potential between two static sources}

The potential at a point $x_{2}$ from a point-like source at $x_{1}$ can be obtained, in the high-temperature limit, by the two-point Green function $G_{2 p}\left(x_{1}-x_{2}\right)$. In the Feynman path-integral formalism $G_{2 p}$ is

$G_{2 p}\left(x_{1}-x_{2}\right)=\frac{1}{Z} \int \mathrm{D} \varphi \varphi\left(x_{1}\right) \varphi\left(x_{2}\right) \mathrm{e}^{-\mathrm{i} S_{\mathrm{s}}}$,

where $S_{\mathrm{S}} \equiv \int \mathrm{d}^{4} x \mathcal{L}$ is the action, $\int \mathrm{D} \varphi$ sums over all possible field configurations, and $Z \equiv \int \mathrm{D} \varphi \mathrm{e}^{-\mathrm{i} S_{\mathrm{s}}}$. On an Euclidean spacetime lattice simulation, the temperature corresponds to inverse of the lattice spacing in the time direction. Hence, in the high-temperature limit, the $\operatorname{sum} \int \mathrm{D} \varphi$ is reduced to configurations in position space. The suppression of time allows us to identify the potential to $G_{2 p}$.

Such expression of the potential is unconventional and restrictive since it applies only to stationary systems. However, its simplicity allows for fast calculations, which in turn may allow the implementation of forces too complex, e.g. gravity with tensor fields, to be efficiently implemented on the lattice in the standard way. Since such a way of calculating potentials is unusual, we will specifically verify its validity for instantaneous potentials, the only cases studied here. This will be demonstrated by correctly recovering potentials in known cases of free-field theories (theories without selfinteracting fields). Although adding terms to the Lagrangian does not affect the above definition of the potential, one might speculate that self-interacting terms may void its validity.
However, we will also recover the known expected potential for the self-interacting $\phi^{4}$ theory as well as the strong interaction phenomenological static potential once short distance quantum effects are accounted for. Based on this we can assume with some confidence that this method of calculating potentials remains valid for the self-interacting field case.

\subsection{Quantum versus classical calculations}

(In this section we keep $\hbar$ in expressions to keep track of quantum effects.) In principle, the path-integral formalism provides intrinsically quantum calculations. However, the results obtained here will be classical since we employ a lattice covering position space only (high-temperature limit) $[9,10]$. Briefly, the system being time-independent, we have $S_{\mathrm{s}} \equiv \int \mathrm{d}^{4} x \mathcal{L}=\tau S$, with $\tau=\int_{t_{0}}^{\infty} \mathrm{d} t \rightarrow \infty$ and $S \equiv \int \mathrm{d}^{3} x \mathcal{L}$. The exponential term in Eq. (4) becomes $\mathrm{e}^{-\mathrm{i} S_{\mathrm{s}} / \hbar}=\mathrm{e}^{-\mathrm{i} \tau S / \hbar}$. Compared to the usual $\mathrm{e}^{-\mathrm{i} S / \hbar}$ exponential in path-integral quantum field theory, quantum effects are suppressed as $\hbar / \tau$. Since $\tau \rightarrow \infty$, this is equivalent to the classical $\hbar \rightarrow 0$ limit.

We remark that our definition of the potential allows one to compute only instantaneous potentials, which are suited for stationary systems and the purpose of the present article. Consequently, computing potentials from the time-propagation of the system, as for example in the Feynman-Kac formula [11,12] or using Wilson loops [13], is not applicable.

In the rest of the paper, we will not write anymore the factor $\tau$ since, as a constant rescaling factor, it is irrelevant to classical calculations (a rescaled extremal action remains extremal or, equivalently, rescaling $S$ amounts to rescaling $\mathcal{L}$, whose rescaling factor cancels out in the Euler-Lagrange Equation). However, $\tau$ must be considered when e.g. discussing the dimensions of the fields and couplings. In other words, the action $S$ has a dimension [ $\hbar \times$ energy] rather than $[\hbar]$.

\subsection{Goal, formalism and verifications}

The goal is to compute the potential between two approximately static $(v \ll c)$ sources in the non-perturbative regime. The lattice technique, based on a standard Monte-Carlo method exploiting the Metropolis algorithm, is described in Appendix A. A practical example is given in Appendix B. In Appendix $\mathrm{C}$, we discuss the delicate point of the choice of boundary conditions and show that the results are independent of such choices. In Appendix D, the method is verified by recovering analytically known free-field potentials in two or three spatial dimensions, as well as the $g^{2} \phi^{4}$ theory potential in three dimensions. In Appendix E, we verify the independence of the results on the choice of lattice parameters. 


\section{Results}

\section{$4.1 g^{2} \phi^{4}$ theory}

The $g^{2} \phi^{4}$ theory can be solved analytically [8]. The analytical solution of the equation of motion is known, with the field obeying a massive dispersion relation. Indeed, the resulting potentials are well fit by a Yukawa potential over the whole range of coupling values we used $\left(0 \leq g^{2} \leq 4\right)$; see Fig. 2 for an example of potential between two sources located at $x_{1}=$ 21 and $x_{2}=35$, and for $g^{2}=0.5$. Figure 2 also demonstrates the independence of the result with the choice of boundary conditions. The expected function describing $\phi$ is the Jacobi elliptic function $\operatorname{sn}(x,-1)$ [8]. Its autocorrelation $\mathrm{sn} \star \mathrm{sn}$, which provides the potential $G_{2 p}$, fits well our numerical results; see Fig. 3, but only for coupling values $g^{2}>1$.

The effect of the quartic term $g^{2} \phi^{4}$ can be embodied using a field effective mass $m_{\text {eff }}$. It can be obtained by fitting $G_{2 p}$ with a Yukawa potential $e^{-m_{\mathrm{eff}} x} / x$. Such effective mass is shown as a function of $g^{2}$ in Fig. 4. A polynomial fit to these data yields $m_{\text {eff }}=(0.76 \pm 0.02)\left(g^{2}\right)^{0.39 \pm 0.02}+$ $0.05 \pm 0.02$, and a logarithmic fit yields $m_{\text {eff }}=(1.13 \pm$ $0.03) \ln \left(\left(g^{2}\right)^{0.51 \pm 0.02}+1\right)+0.06 \pm 0.02$ with a marginally better $\chi^{2}$ (10\% smaller). As already mentioned, the effectively massive behavior of the $g^{2} \phi^{4}$ theory can be understood from the lack of partial derivatives in the quartic term. It thus does not contribute to the field propagation and is akin to a mass term. Evidently, this field effective mass depends on the value of the coupling $g^{2}$ (Fig. 4).

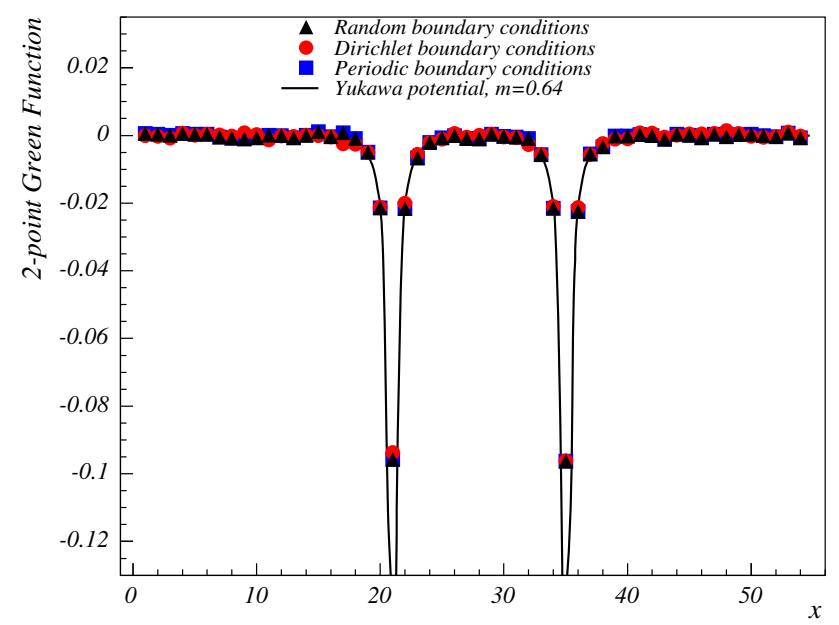

Fig. 2 Potential $G_{2 p}$ for the $g^{2} \phi^{4}$ theory, plotted as a function of distance. The simulations used a lattice size $N=55$, a coupling $g^{2}=0.5$, a decorrelation coefficient (see Sect. B.1) $N_{\text {cor }}=20$ and $N_{\mathrm{s}}=5 \times 10^{4}$ configurations. The results are independent of the choice of boundary conditions. They are well fit by two Yukawa potentials centered on each two sources at $d= \pm 7$ from the lattice center and with a mass parameter $m_{\text {eff }}=0.64$ (continuous line)

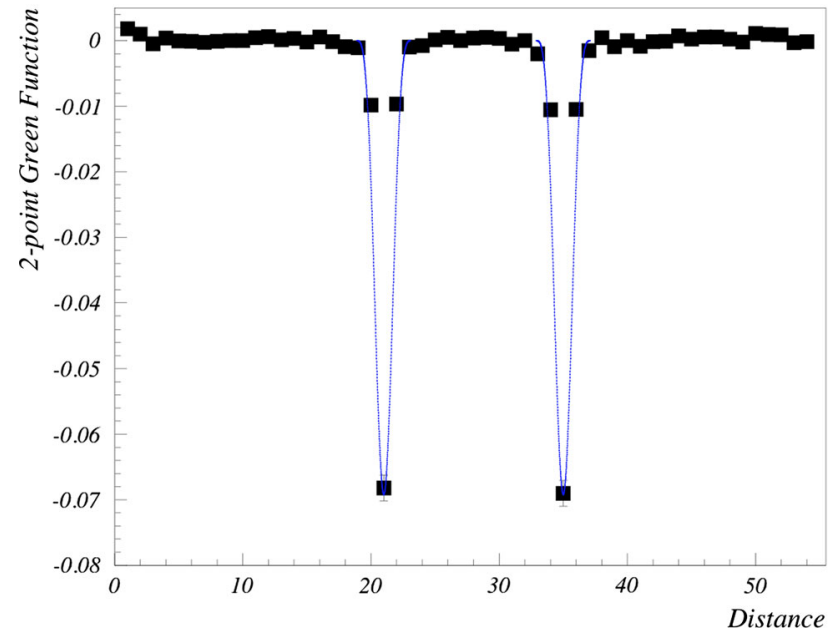

Fig. 3 Comparison between the potential computed numerically using Eq. (1) (squares) and the analytical expectation $\operatorname{sn}(x,-1) \star \operatorname{sn}(x,-1)$ (line). The simulation used a coupling $g^{2}=3$, a lattice size $N=55$, $N_{\mathrm{s}}=5 \times 10^{4}$ configurations, a decorrelation coefficient $N_{\text {cor }}=15$ and random field boundary conditions

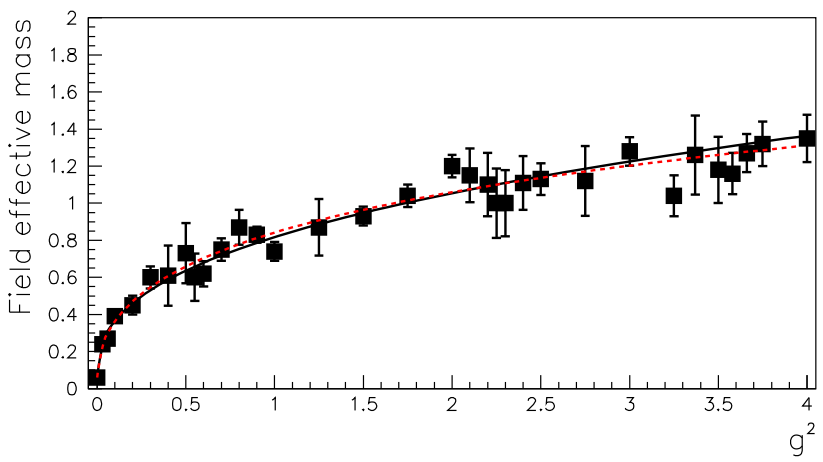

Fig. 4 The $g^{2}$-dependence of the effective mass generated by the quartic term $g^{2} \phi^{4}$. The continuous line is a polynomial fit to the data and the dashed line is a logarithmic fit. The simulation used a lattice size $N=55, N_{\mathrm{s}}=5 \times 10^{4}$ configurations, a decorrelation coefficient $N_{\text {cor }}=15$ and random field boundary conditions

\section{$4.2\left(g \phi \partial_{\mu} \phi \partial^{\mu} \phi+g^{2} \phi^{2} \partial_{\mu} \phi \partial^{\mu} \phi\right)$ theory}

The $\left(g \phi \partial_{\mu} \phi \partial^{\mu} \phi+g^{2} \phi^{2} \partial_{\mu} \phi \partial^{\mu} \phi\right)$ theory in its nonperturbative regime yields the static potential shown in Fig. 5. It varies approximately linearly with distance (except at middistance between the two sources where the potential flattens out, as expected for a solely attractive force). Also shown is the $g=0$ case which yields the expected free massless field potential in $1 / x$, with $x$ the distance to one of the source. In Fig. 6, results are shown with the $1 / x$ contribution subtracted in order to isolate the linear part stemming from the $\left(g \phi \partial_{\mu} \phi \partial^{\mu} \phi+g^{2} \phi^{2} \partial_{\mu} \phi \partial^{\mu} \phi\right)$ term.

The linear $x$-dependence of the potential can be interpreted in a similar manner as done in the QCD static case; see e.g. [1]. In QCD, the linear potential is often pictured as originating from the collapse of the force's field lines into 


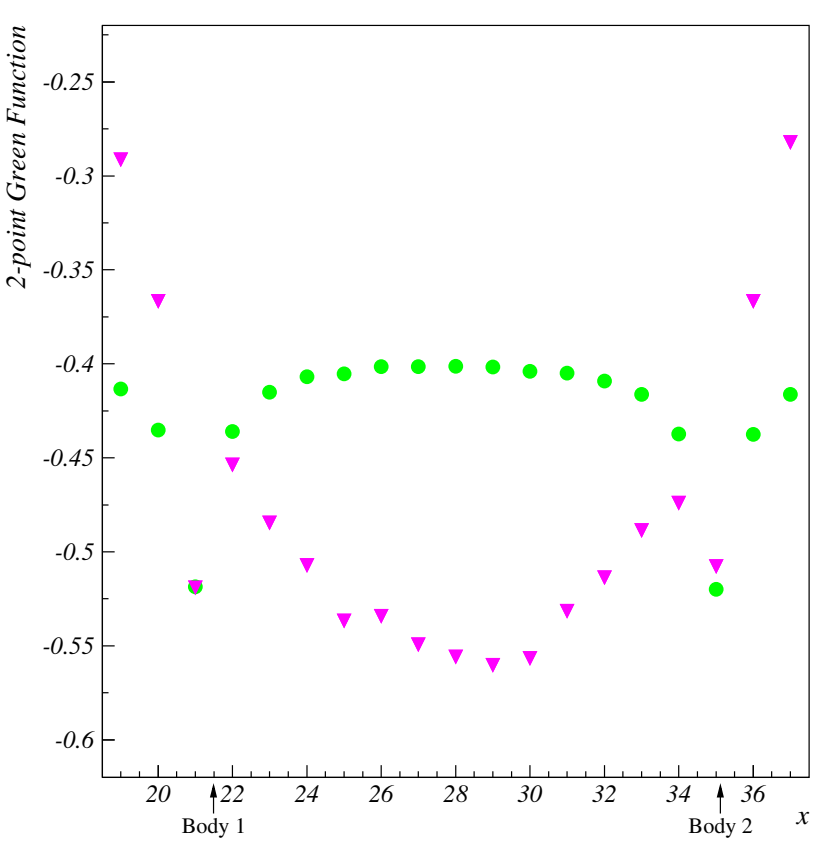

Fig. 5 The potential for the $\left(g \phi \partial_{\mu} \phi \partial^{\mu} \phi+g^{2} \phi^{2} \partial_{\mu} \phi \partial^{\mu} \phi\right)$ theory ( $t r i-$ angles). The circles are the free-field case $(g=0)$. The two sources are located on the $x$-axis at $d= \pm 7$ from the lattice center $x_{\text {center }}=28$, $y=0$ and $z=0$. The coupling is $g=7.5 \times 10^{-3}$, the lattice size is $N=85$, the decorrelation parameter is $N_{\text {cor }}=20$ and $N_{\mathrm{s}}=3.5 \times 10^{4}$ paths were used. To conveniently compare the results, a constant offset is added to the free-field case so that the potentials at the position of the first source, $x=21$, match

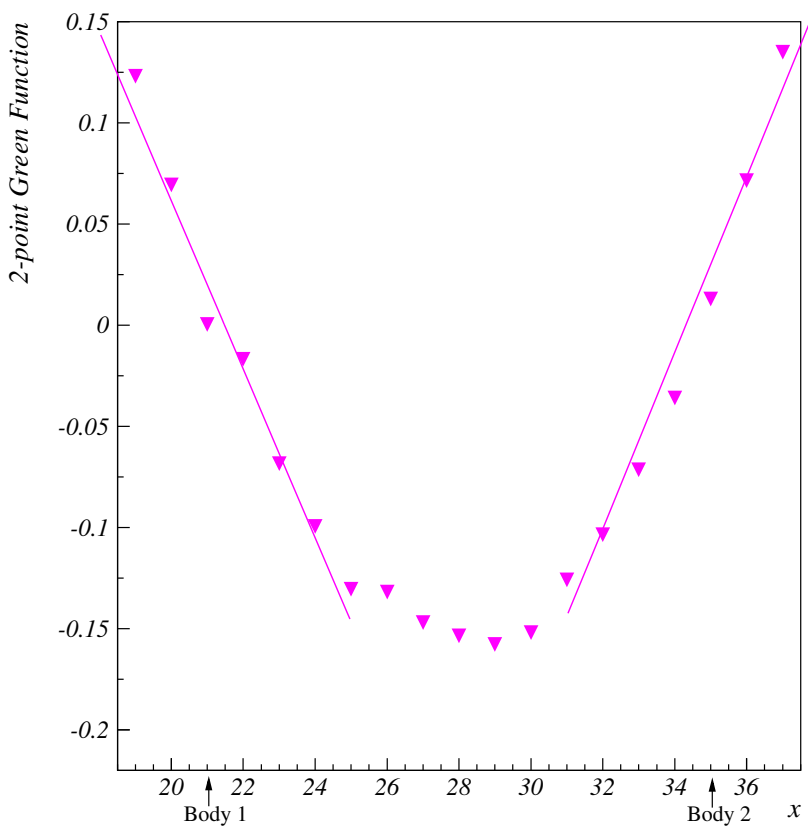

Fig. 6 Same as Fig. 5 but with the $1 / x$ - free-field - contribution subtracted. The lines illustrate the nearly linear trend of the potential

a flux tube linking the two sources. This collapse is caused by strong non-perturbative field self-interactions: the gluons strongly attract each other toward the region of their highest density i.e. the segment joining the sources. If the sources are static and since the field has collapsed into a segment, the two dimensions transverse to the segment become irrelevant. The strong non-perturbative effects have constrained the field flux from three spatial degrees of freedom to one. The resulting force is constant, as easily calculated or visualized with Faraday's flux picture.

A similar phenomenon seems to occur with the theory using $\left(g \phi \partial_{\mu} \phi \partial^{\mu} \phi+g^{2} \phi^{2} \partial_{\mu} \phi \partial^{\mu} \phi\right)$ theory. The strong field self-interaction effectively reduces the three-dimensional system to a one-dimensional system, yielding a linear potential. The linear potential can also be understood from field correlations. The potential is given by the two-point Green function, i.e. the autocorrelation function of the field; see Eq. (4). It is a general property that the autocorrelation of the sum of two uncorrelated functions is the sum of the autocorrelations of each function separately: if $f=g+h$, then $f \star f=g \star g+h \star h$, i.e. two uncorrelated fields $g$ and $h$ (i.e. $g$ and $h$ do not influence each others) from two sources imply the superposition principle. The total potential $f \star f$ is the sum of the potentials $g \star g$ and $h \star h$. If the fields mutually influence each others, correlations appear. The superposition principle is thus broken. When fields are interacting strongly enough, they become strictly correlated. The total field (observed sufficiently away from the sources so that the three-dimensional symmetry around a point-like source is broken) is then constant on a segment linking the two sources. It must quickly go to zero outside the segment due to the boundary conditions at infinity. The field is then approximately a one-dimensional rectangular function. The autocorrelation of a rectangular function being a triangle function, the potential between the two sources has a linear $x$-dependence, as seen in Fig. 6. In all, the linear potential arises due to the long field autocorrelation length - stemming from the partial derivatives present in each term of the Lagrangian - that couples the field at different locations in space. This explanation is consistent with the naive dimensional argument inspired from QCD.

With this interpretation, we can immediately generalize the present two-source linear potential result to a uniform and homogeneous disk of sources. The three-dimensional system is then reduced to two dimensions. This yields a logarithmic potential (see Fig 12). For a uniform and homogeneous spherical distribution of sources, the system stays three-dimensional and retains a static $1 / x$ potential.

\section{Connections to QCD and GR}

In this section, we explore the possible connections of the Lagrangians in Eqs. (1) and (2) with QCD and GR, respectively. We first discuss the benefits that such connections would provide. 
Numerical studies of QCD using lattice gauge theory are sophisticated and well checked. They have provided invaluable information on this theory. As stated in the introduction, the present work is not meant to compete with these more exact and more mature approaches. The primary goal of making the connection to QCD is that, if the QCD phenomenology is recovered, it would support the validity of the method beyond the checks already discussed. The method can then be used with confidence to address more complicated cases, e.g. GR whose tensorial field makes it currently impractical to put on the lattice. This primary goal being clarified, there are still advantages of such approach within the sole context of the strong interaction: (1) a simple approach showing the onset of confinement may isolate its essential aspects and make them easier to study, including analytically. Furthermore, (2) if the confinement mechanism is manifest in a simplified approach, it may provide guidances for an analytical resolution of the problem. It could (3) also provide an alternate approach to a complex problem. In fact, lattice QCD has greatly benefited from other nonperturbative approaches that can be fully solved only numerically, e.g. the Schwinger-Dyson equations approach. (4) It makes calculations fast compared to typical lattice running time - a few hours of running on a standard personal computer are sufficient. (5) It provides a pedagogical model of confinement.

Efforts to solve GR in its non-perturbative regime by numerical methods also constitute an active field of study [14]. Its object is strongly interacting systems for which gravitational backreaction cannot be ignored. As in QCD, the equations governing these systems are non-linear and can be non-perturbative. Except in rare cases, they can only be solved numerically. A non-perturbative component to the potential would not be identified using the usual perturbative (post-Newtonian) approximation, and a non-perturbative linear contribution to the potential is in general compatible with the field equations of bound systems $[15,16]$. In fact, it is emphasized in Refs. $[15,16]$ that bound states can be understood semi-classically as divergences of the perturbative expansion arising from ladder-type Feynman diagrams. Consequently, even more than for $\mathrm{QCD}$, possessing alternate methods to approach the complex problem of bound systems in GR is beneficial.

In the rest of this section, we discuss the possibility of such method, based on the fact that the Lagrangians in Eqs. (1) and (2) may be related to the QCD and GR Lagrangians. Such relation has already been put forward in the case of QCD $[17,18]$ where it was asserted that Eq. (1) is equivalent to QCD in either the classical limit or the low energy limit. We will show that similarly, Eq. (2) may describe GR in the classical limit. We first start by recalling the Lagrangians of QCD and GR.

\subsection{QCD Lagrangian}

The QCD field Lagrangian is

$$
\begin{aligned}
\mathcal{L}_{\mathrm{QCD}}^{\mathrm{field}}= & -\frac{1}{4} F_{\mu \nu}^{a} F_{a}^{\mu \nu} \\
= & \frac{1}{4}\left(\partial_{\nu} A_{\mu}^{a}-\partial_{\mu} A_{\nu}^{a}\right)\left(\partial^{\mu} A^{v a}-\partial^{v} A^{\mu a}\right) \\
& +\sqrt{\pi \alpha_{s}} f^{a b c}\left(\partial_{\nu} A_{\mu}^{a}-\partial_{\mu} A_{\nu}^{a}\right) A^{\mu b} A^{v c} \\
& -\pi \alpha_{s} f^{a b e} f^{c d e} A_{\mu}^{a} A_{\nu}^{b} A^{\mu c} A^{v d}
\end{aligned}
$$

with $\alpha_{s}$ the QCD coupling constant, $A_{\mu}^{a}$ the gluon field and with the SU(3) index $a=1, \ldots, 8$. We do not include Fadeev-Popov ghosts in the Lagrangian. Their necessity depends on the choice of gauge, e.g. there are no ghosts in the axial gauge $e^{\mu} A_{\mu}^{a}$ or in the light-cone gauge $A^{+}=0$. Furthermore, Fadeev-Popov ghosts are needed in QCD essentially to force the gluon propagator to remain transverse when loop diagrams are included and since we are discussing here limits using spinless fields, there is no such need. Fermions are also not included in Eq. (5) since it is sufficient to work in the pure field sector for a first study of confinement in the static case.

\subsection{General relativity}

Einstein's field equations of GR are generated by the Einstein-Hilbert Lagrangian density:

$\mathcal{L}_{\mathrm{GR}}=\frac{1}{16 \pi G} \sqrt{\operatorname{det}\left(g_{\mu \nu}\right)} g_{\mu \nu} R^{\mu \nu}$,

where $G$ is the Newton constant, $g_{\mu \nu}$ the metric and $R_{\mu \nu}$ the Ricci tensor. The gravity field $\varphi_{\mu \nu}$ is defined as the difference between $g_{\mu \nu}$ and a constant reference metric $\eta_{\mu \nu}$ : $\varphi_{\mu \nu} \equiv\left(g_{\mu \nu}-\eta_{\mu \nu}\right) / \sqrt{16 \pi G}$. Here, $\eta_{\mu \nu}$ will be the flat metric. Expanding $\mathcal{L}_{\mathrm{GR}}$ in terms of $\varphi_{\mu \nu}$ yields (see e.g. [19] or [20]):

$$
\begin{aligned}
& \mathcal{L}_{\mathrm{GR}}=[\partial \varphi \partial \varphi]+\sqrt{16 \pi G}[\varphi \partial \varphi \partial \varphi]+16 \pi G\left[\varphi^{2} \partial \varphi \partial \varphi\right] \\
& \quad+\cdots-\sqrt{16 \pi G} \varphi_{\mu \nu} T^{\mu \nu}-\frac{16 \pi G}{2} \varphi_{\mu \nu} \varphi_{\sigma \lambda} T^{\mu \nu} \eta^{\sigma \lambda}+\cdots,
\end{aligned}
$$

where $T^{\mu \nu}$ is the energy-momentum tensor and $\left[\varphi^{n} \partial \varphi \partial \varphi\right]$ is a shorthand notation for a sum over the possible Lorentz invariant terms of the form $\varphi^{n} \partial \varphi \partial \varphi$. For example, $[\partial \varphi \partial \varphi]$ is explicitly given by the Fierz-Pauli Lagrangian [21], the first order approximation of GR that leads to Newton's gravity:

$$
\begin{aligned}
{[\partial \varphi \partial \varphi]=} & \frac{1}{2} \partial^{\lambda} \varphi_{\mu \nu} \partial_{\lambda} \varphi^{\mu \nu}-\frac{1}{2} \partial^{\lambda} \varphi_{\mu}^{\mu} \partial_{\lambda} \varphi_{\nu}^{\nu} \\
& -\partial^{\lambda} \varphi_{\lambda \nu} \partial_{\mu} \varphi^{\mu \nu}+\partial^{\nu} \varphi_{\lambda}^{\lambda} \partial^{\mu} \varphi_{\mu \nu} .
\end{aligned}
$$

Equation (7) is expanded in term of the dimensionless quantity $\sqrt{16 \pi G} \varphi$. It is justified to truncate Eq. (7), even 
for strong gravity fields, since in general $\sqrt{16 \pi G} \ll 1 / \mu$ with $\mu$ the typical mass scale or inverse distance scale of the system considered. A pertinent reference metric $\eta_{\mu \nu}$ is chosen according to the system being considered. It can be e.g. the Minkowski metric for systems inducing weak curvatures, or the Schwarzschild metric for a system involving a black hole. Hence, Eq. (7) is applicable to both the weak-field approximations of GR and non-perturbative gravity.

\subsection{Lagrangians in the static case}

Equation (7) can be simplified. Consider first the $[\partial \varphi \partial \varphi]$ term given by Eq. (8). The Euler-Lagrange equation obtained by varying $\varphi_{\mu \nu}$ in $\int \mathrm{d}^{4} x\left([\partial \varphi \partial \varphi]-\sqrt{16 \pi G} \varphi_{\mu \nu} T^{\mu \nu}\right)$ leads to $\partial^{2} \varphi^{\mu \nu}=-16 \pi G\left(T^{\mu \nu}-\frac{1}{2} \eta^{\mu \nu} \operatorname{Tr} T\right)$. Since $T^{00}$ dominates over the other components of $T^{\mu \nu}$ in the static limit, $\partial^{2} \varphi^{\mu \mu} \gg \partial^{2} \varphi^{\mu \nu}$ for $\mu \neq v$ and $\partial^{2} \varphi^{00} \simeq \partial^{2} \varphi^{i i}$. Thus, $\varphi^{00}=\varphi^{i i}+a_{i} x+b_{i}$. Since $\varphi^{\mu \nu} \rightarrow 0$ for $x \rightarrow \infty$, $a_{i}=0, b_{i}=0$ and $\varphi^{00}=\varphi^{i i}$. Replacing the $\varphi^{i i}$ terms in Eq. (8) by $\varphi^{00}$ and applying the harmonic gauge condition $\partial^{\mu} \varphi_{\mu \nu}=\frac{1}{2} \partial_{\nu} \varphi_{\kappa}^{\kappa}$, leads to $[\partial \varphi \partial \varphi]=\partial_{\lambda} \varphi^{00} \partial^{\lambda} \varphi_{00}$. Consider now the next order term, $[\varphi \partial \varphi \partial \varphi]$. The $\varphi^{\mu \nu}(\mu \neq \nu)$ terms that were neglected in the lower order operations leading to $[\partial \varphi \partial \varphi]=\partial_{\lambda} \varphi^{00} \partial^{\lambda} \varphi_{00}$ can be ignored with respect to the $\varphi^{00} \partial_{\lambda} \varphi^{00} \partial^{\lambda} \varphi^{00}$ term: the terms [ $\partial \varphi \partial \varphi$ ] lead to linear field equations, so they do not contribute to the non-linearity due to the $\left[\varphi^{n} \partial \varphi \partial \varphi\right]$ terms, while they remain negligible compared to the linear terms $\partial_{\lambda} \varphi^{00} \partial^{\lambda} \varphi^{00}$. The $\partial_{\lambda} \varphi^{00} \partial^{\lambda} \varphi_{00}$ structure dominates all other components of the form $[\partial \varphi \partial \varphi]$. Thus, if the influence of the $\varphi^{00} \partial_{\lambda} \varphi^{00} \partial^{\lambda} \varphi^{00}$ term is not negligible compared to the effect of the $\partial_{\lambda} \varphi^{00} \partial^{\lambda} \varphi^{00}$ term (i.e. there are strong field non-linearities) then it is justified to neglect the components $\varphi^{\mu \nu}(\mu \neq v)$ of $[\partial \varphi \partial \varphi]$. The corrections coming from the modification of the Euler-Lagrange equation enter only at the level of the terms $\varphi^{n+1} \partial^{2} \varphi$, with $n \geq 2$, and can likewise be neglected. Consequently, in the static case which identifies to the high-temperature limit in Euclidean space -, the GR Lagrangian may be simplified to

$\mathcal{L}_{\mathrm{GR}, \text { stat. }}=\sum_{n=0}^{\infty}(16 \pi G)^{n / 2} \varphi^{n}\left[a_{n} \partial \varphi \partial \varphi-T_{00}\right]$

where the subscript stat. reminds us that $\mathcal{L}_{\mathrm{GR}}$ is expressed in the static limit. We define the scalar field $\varphi \equiv \varphi_{00}$. We found above that $a_{0}=1$ and confirm it in Appendix $\mathrm{F}$ by deriving the weak-field potential from Eq. (9), and comparing it to the Einstein-Infeld-Hoffmann equations [22]. The values of the other $a_{n}$ coefficients are not important here since Eq. (9) will be used only at first orders. We set $a_{n}=1$ for all $n$ in the rest of the paper. For two static point sources located at $x_{1}$ and $x_{2}, \mathcal{L}_{\mathrm{GR}, \text { stat. }}$ becomes

$$
\begin{aligned}
& \mathcal{L}_{\mathrm{GR}, \text { stat. }}=\sum_{n=0}^{\infty}(16 \pi G)^{n / 2}\left[\varphi^{n} \partial \varphi \partial \varphi\right. \\
& \left.-(16 \pi G)^{1 / 2} \varphi^{n+1}\left(\delta^{(4)}\left(x-x_{1}\right)+\delta^{(4)}\left(x-x_{2}\right)\right)^{n+1}\right] .
\end{aligned}
$$

Gravitation is a spin-2 theory and, like any spin-even theory, is always attractive. Satisfactorily, the scalar, spin- 0 , approximation is also spin-even and thus also always attractive. In all, we see that the pure field part of $\mathcal{L}_{\mathrm{GR}}$,stat. is identical to Eq. (2), thus supporting that GR can be adequately described in its static limit with the Lagrangian in Eq. (2).

Likewise in QCD, the chromoelectric component $A_{0}^{a} \equiv A$ of the gluon field dominates in the case of two static sources and the QCD Lagrangian may be approximated by

$\mathcal{L}=\partial_{\mu} A \partial^{\mu} A-\pi \alpha_{s} A^{4}$.

Contrary to Eq. (5), Eq. (11) has no cubic term. In the strong coupling regime where non-perturbative effects are dominant, either $\pi \alpha_{S} A^{4} \gg \partial A \partial A$ if only the quartic term dominates in Eq. (5), or $\sqrt{\pi \alpha_{S}} A^{2} \gg \partial A$ if the cubic term dominates over the quadratic one. The latter inequality also implies $\sqrt{\pi \alpha_{S}} A^{4} \gg A^{2} \partial A$ so in any case, the quartic term dominates over the other terms. Thus, the lack of a cubic term in Eq. (11) may not preclude that this equation describes QCD in a classical limit. In fact, it has been advanced that Eq. (11) describes QCD in either the classical limit when quantum fluctuations are neglected, or in the low energy, strong coupling, limit [17,18].

In QCD, a two-source system corresponds to a meson which, to be colorless and have zero baryon number, must be made of constituent quark and antiquark of opposite colors. QCD being a spin-1 theory, the force either attracts or repulses. However, the force between the two static sources is solely attractive since they must carry opposite colors. Hence, as for gravity, the always attractive spin-0 field is also satisfactory in the case of strong interaction. This also applies to baryons when viewed as quark-diquark structures.

In all, the QCD and GR Lagrangians, Eqs. (5) and (7), respectively, may be approximated in the classical and static limits by the scalar Lagrangians of Eqs. (11) and (9), respectively. We remark that the invariance principles underlying the theories, e.g. gauge invariance under $\mathrm{SU}(3)_{c}$ for QCD, cannot be considered in scalar theories. However, they are manifest in the sense that the form of the scalar Lagrangians stem from the ones of the full theories whose Lagrangian forms themselves are imposed by invariance principles. Likewise, the non-abelian nature of the theories cannot be formalized with a scalar theory but is still manifest in the non-linear terms of the Lagrangian. Related features that the full theories possess, e.g. different colors charges, must be accounted for ad hoc, e.g. by inserting the appropriate Casimir factor in the static potential. 


\subsection{Onset of the strong regime for QCD and GR}

The QCD coupling at long distance is large [23]. There, effects from the higher order cubic and quartic terms of Eq. (5) become dominant and quarks are confined. In the gravitational force case, what can lead to a non-perturbative regime is either interactions at very short distances (presumably described by quantum gravity, which we do not consider here), or a large system mass $M$, which is what concerns us here. The gravitational force is a function of its total field squared, $\varphi^{2}$ and is also proportional to $M$. Normalizing $\varphi$ to $\varphi^{2}=M \phi^{2}$ makes explicit the importance of the higher order terms $(16 \pi G)^{n / 2} M^{n / 2+1} \varphi^{n} \partial \varphi \partial \varphi$ in Eq. (10) for large values of $M$. Rescaling $\mathcal{L}$ by $1 / M$, the three-dimensional action based on Eq. (10) is

$$
\begin{aligned}
S= & \int \mathrm{d}^{3} x\left[\partial_{i} \phi \partial^{i} \phi+g \phi \partial_{i} \phi \partial^{i} \phi+g^{2} \phi^{2} \partial_{i} \phi \partial^{i} \phi+\cdots\right. \\
& \left.-g \phi\left(\delta^{(3)}\left(x-x_{1}\right)+\delta^{(3)}\left(x-x_{2}\right)\right)-\cdots\right],
\end{aligned}
$$

with $g=\sqrt{16 \pi M G}$. (The integration of the Lagrangian density being here three-dimensional; see Sect. 3.1, we summed over the spatial index $i$ rather than the Lorentz index $\mu$.) We note that rescaling thus the Lagrangian emphasizes further the quantum effect suppression discussed in Sect. 3.1. In all, quantum effects for the theory given by Eq. (10) are suppressed by $\hbar / M \tau$, with $M$ a large mass in our regime of interest.

In QCD, the non-linear regime arises for distances greater than $2 \times 10^{-16} \mathrm{~m}$ when $\alpha_{s}$ becomes large: $\alpha_{s} \simeq 0.5$ in the $\overline{M S}$ scheme [23]. Likewise, such a regime should arise in GR when $g=\sqrt{16 \pi G M}$ is large, with $M$ the system mass, and the system size is comparable to the field correlation length $L$. For a galaxy, typically $\sqrt{16 \pi G M / L} \simeq 10^{-2}$, with $L=10 \mathrm{kpc}$ taken to be a third of a typical galaxy size, and $M=3 \times 10^{11} M_{\odot}$, a typical galaxy mass. $g \simeq 10^{-2}$ is large enough to trigger the non-linear regime onset; see Fig. 5.

\subsection{Applying the scalar model to QCD and GR in the high-temperature limit}

\subsection{1 $Q C D$}

While GR is a classical theory, and thus relevant for comparison with results obtained using the Lagrangian in Eq. (10), there is a priori no well-known classical limit pertinent to QCD, although semi-classical approximations do exist $[15,16,24]$. Certainly, an approximate Yukawa potential such as the one obtained in the high-temperature limit for the $g^{2} \phi^{4}$ theory; see Fig. 2, does not resemble the linear potential expected in the QCD static case. This indicates that, provided Eq. (1) indeed describes the classical aspect

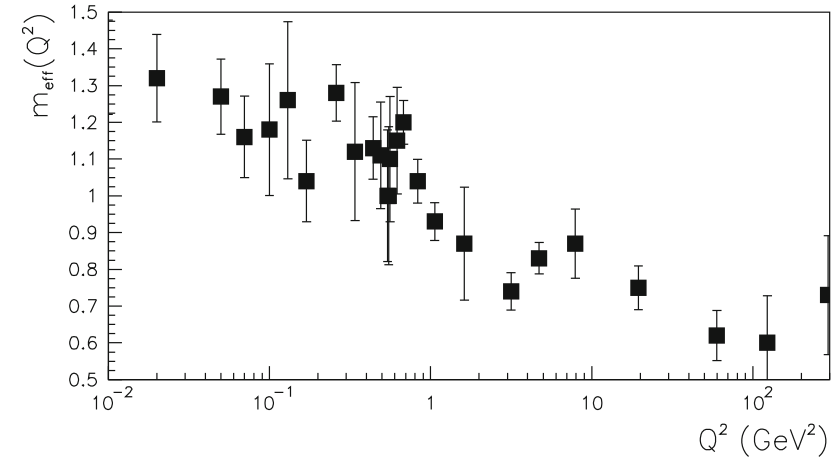

Fig. 7 The running effective mass $m_{\mathrm{eff}}\left(Q^{2}\right)$ as a function of the 4momentum transfer $Q^{2}$ corresponding to the $g^{2}$ values shown in Fig. 4

of QCD, quantum effects are essential for obtaining a linear potential. Whereas, in the pure field sector of $\mathrm{QCD}$, quantum effects such as quark condensates are irrelevant, others such as the running of the coupling cannot be disregarded when comparing meaningfully with phenomenology. Accounting for it can be done by mapping the $g^{2}$-dependence of $m_{\mathrm{eff}}$ into a 4-momentum transfer $Q^{2}$-dependence assuming that $g^{2} / \pi$ runs as the QCD coupling $\alpha_{s}\left(Q^{2}\right)$ [23]. (Equations (1) and (5) allow us to identify $g^{2} / \pi$ to $\alpha_{s}$.) By augmenting the $g^{2} \phi^{4}$ theory with the running of the coupling, we introduce short-distance quantum effects in an otherwise classical setting. We used the AdS/QCD results on $\alpha_{s}\left(Q^{2}\right)$ [25-27] for $Q^{2} \lesssim 1 \mathrm{GeV}^{2}$, supplemented by the perturbative QCD expression of $\alpha_{S}\left(Q^{2}\right)$ at fourth order for larger $Q^{2}$. Such $\alpha_{s}\left(Q^{2}\right)$ agrees well with the phenomenology $[28,29]$ for all $Q^{2}$. Specifically, we used $\alpha_{s}\left(Q^{2}\right)$ in the conventional $\overline{M S}$ renormalization scheme. The result is shown in Fig. 7.

Using these results, the potential of the $g^{2}(x) \phi^{4}$ theory becomes

$G_{2 p}(x)=-C_{f} \frac{g^{2}(x)}{\pi} \frac{\mathrm{e}^{-m_{\mathrm{eff}}(x) x}}{x}$,

where we included the appropriate color factor $C_{f}=4 / 3$ for a more pertinent comparison with $\mathrm{QCD}$, and where $g^{2}\left(Q^{2}\right)$ and $m_{\text {eff }}\left(Q^{2}\right)$ were Fourier-transformed to position space. The result is shown in Fig. 8 and compared with the well-established Cornell potential [30-34] that successfully describes hadron spectroscopy and that is consistent with quenched SU(3) QCD lattice calculations [35]. $G_{2 p}(x)$ displays the expected linear behavior in the relevant $x$ range and agrees well with the Cornell potential. (For this comparison, $x$ in Eq. (13) must be rescaled by $1 / C_{f}$, since not having color factors in Eq. (11) underestimates the magnitude of the field coupling by $C_{f}$ compared to the SU(3) QCD theory.) The string tension, fit from the results between 0.35 and 0.6 fm in Fig. 8, is $\sigma=0.157 \pm 0.012 \mathrm{GeV}^{2}$. This yields a con- 


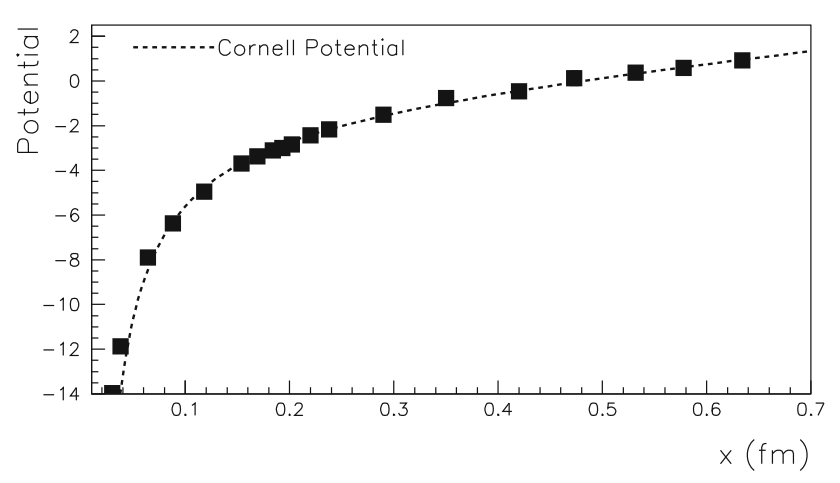

Fig. 8 High-temperature potential from the $g(x)^{2} \phi^{4}$ theory as a function of distance after adding the runnings of $g^{2}$ and of $m_{\mathrm{eff}}$. The strong interaction's Cornell potential is shown by the dashed line

finement parameter $\kappa=\sqrt{\sigma \pi / 2}=0.496 \pm 0.038 \mathrm{GeV}$ that agrees with the value $\kappa=0.523 \pm 0.024 \mathrm{GeV}$ found in [3].

The appearance of a mass scale in the $g^{2} \phi^{4}$ theory, despite its conformal Lagrangian, is a valuable feature. Understanding the emergence of a $\mathrm{GeV}$-size mass scale is one of the outstanding problems of strong interaction; see e.g. [24]. In the present work, such emergence can be understood from the absence of derivatives in the quartic term $g^{2} \phi^{4}$, which thus does not contribute to field propagation but acts as a field mass. QCD also possesses a non-propagating $A_{\mu}^{a} A_{v}^{b} A^{\mu c} A^{v d}$ term in its Lagrangian. This suggests that the mass scale emergence in strong interaction is a classical effect due to the specific form of the quartic term of the QCD Lagrangian. The cubic term may contribute similarly, or may instead generate a long correlation length as it does in the GR case. Although the good agreement of the $g^{2}(x) \phi^{4}$ theory with strong interaction suggests a minor role of the cubic term, its actual effect cannot be explored in the present model.

In all, the running effective mass and the potential obtained from the Lagrangian in Eq. (11) are consistent with what is expected from QCD. If this consistency underlines the pertinence of the $g(x)^{2} \phi^{4}$ theory to model QCD, then it indicates that quantum effects, responsible for the running of the strong coupling, play a role in shaping the potential. Augmenting the $g^{2} \phi^{4}$ theory at high-temperature with the running of $g^{2}$ is necessary to map the values of the field's effective mass into a $Q^{2}$-running mass, and to change the Yukawa potential into a linear potential. (An equivalent solution leading to a linear potential without introducing a running of the coupling would be to express the dressed gluon propagator as a sum of massive field propagators as done in Refs. [17,18,36].) A consistency between the $g^{2}(x) \phi^{4}$ theory and QCD also encourages the application of the present approach to the more complex case of GR, a fortiori since GR is already a classical theory and would not need to be supplemented ad hoc by quantum effects.

\subsubsection{General relativity}

The $\left(g \phi \partial_{\mu} \phi \partial^{\mu} \phi+g^{2} \phi^{2} \partial_{\mu} \phi \partial^{\mu} \phi\right)$ theory calculations in the high-temperature limit yield a potential which varies approximately linearly with distance; see Figs. 5 and 6.

This can be pictured as a collapse of the three-dimensional system into one dimension. As discussed in Sect. 5.4 and shown numerically, typical galaxy masses are enough to trigger the onset of the strong regime for GR.

Hence, for two massive bodies, such as two galaxies or two galaxy clusters, this would result in a string containing a large gravity field that links the two bodies - as suggested by the map of the large structures of the universe. That this yields quantitatively the observed dark mass of galaxy clusters and naturally explains the Bullet Cluster observation [7] was discussed in [37].

For a homogeneous disk, the potential becomes logarithmic. Furthermore, if the disk density falls exponentially with the radius, as it is the case for disk galaxies, it is easy to show that a logarithmic potential yields flat rotation curves: a body subjected to such a potential and following a circular orbit (as stars do in disk galaxies to good approximation) follows the equilibrium equation:

$v(r)=\sqrt{G^{\prime} M(r)}$,

with $v$ the tangential speed and $M(r)$ the disk mass integrated up to $r$, the orbit radius. $G^{\prime}$ is an effective coupling constant of dimension $\mathrm{GeV}^{-1}$ similar to the effective coupling $\sigma$ (string tension) in QCD. Disk galaxies density profiles typically fall exponentially: $\rho(r)=M_{0} \mathrm{e}^{-r / r_{0}} /\left(2 \pi r_{0}^{2}\right)$, where $M_{0}$ is the total galactic mass and $r_{0}$ is a characteristic length particular to a galaxy. Such a profile leads to, after integrating $\rho$ up to $r$ :

$v(r)=\sqrt{G^{\prime} M_{0}\left(1-\left(r / r_{0}+1\right) \mathrm{e}^{-r / r_{0}}\right)}$,

At small $r$, the speed rises as $v(r) \simeq \sqrt{G^{\prime} M_{0}} r / r_{0}$ and flatten at large $r: v \simeq \sqrt{G^{\prime} M_{0}}$. This is what is observed for disk galaxies and the present approach yields rotation curves agreeing quantitatively with observations [37]. Flat rotation curves are an essential feature of galaxy dynamic that is natural in the present approach. In contrast, they are implemented in an ad hoc way in the standard WIMP/axion approach to dark matter, the profile of the dark matter halo being chosen specifically to yield flat rotation curves.

A problematic observation for non-baryonic dark matter is the correlation between the rotation speeds and baryonic masses of disk galaxies, a phenomenon known as the TullyFisher relation $[4,5]$. The relation is puzzling since it is a dynamical relation that does not involve the galaxy dark mass while this one dominates the total galaxy mass. We showed in [37] how this relation is also naturally explained from the logarithmic potential arising in a disk galaxy. This mass- 
rotation relation of GR can be paralleled to the mass-angularmomentum relation of QCD at the origin of the Regge trajectories $[1,38]$. The two relations are strikingly similar.

For a uniform and homogeneous spherical distribution of matter, the system remains three-dimensional and the static potential stays proportional to $1 / r$, in contrast to the linear or logarithmic potentials of the one- and two-dimensional cases, respectfully. The dependence of the potential with the system's symmetry suggested a search for a correlation between the shape of galaxies and their dark masses [37]. Evidence for such correlation has been found [6].

\section{Summary and conclusion}

We have numerically studied non-linearities in two particular scalar field theories. In the high-temperature limit these may provide a possible description of the strong interaction and of General Relativity (GR) that would simplify their study in their non-perturbative regime, and allow for fast numerical calculations. The overall validity of the method is verified by recovering analytically known potentials. We further verified the validity of the simplifications in the case of GR by recovering the post-Newtonian formalism.

Several approaches to QCD in its non-perturbative regime already exist, such as e.g. Lattice Gauge Theory, DysonSchwinger Equations, Functional Renormalization Group, Stochastic Quantization, or the AdS/CFT correspondence to name a few. They are more advanced and more exact that the simplified method discussed here. What primarily justifies the development of this simpler approximation to QCD is that it provides further checks of the method, which we can then be applied with confidence to the GR case. A subsidiary benefit of testing the method with QCD is that it may help to isolate important ingredients leading to confinement. In that context, this method is able to provide: (1) the expected field function, (2) a mechanism - straightforwardly applicable to QCD - for the emergence of a mass scale out of a conformal Lagrangian, (3) a running of the field effective mass, and (4) a potential agreeing with the phenomenological Cornell linear potential up to $0.8 \mathrm{fm}$, the relevant range for hadronic physics. Quantum effects, which cause couplings to run, are necessary for producing the linear potential and must be supplemented to the approach. A non-running coupling, even with a large value, would only yield a short range Yukawa potential. That the method recovers the essential features of QCD supports its application to GR. Since GR is a classical theory, no running coupling needs to be supplemented. The main goal of the paper is to provides a simple but reliable method to study GR in its non-perturbative regime since compared to QCD, non-perturbative methods for GR are not as developed. It is interesting to develop a method for GR that relates to QCD's formalism since QCD and GR share similar field
Lagrangians, with cubic and quartic field self-interactions terms, and since - contrary to GR - QCD's non-perturbative phenomenology is well studied both experimentally and theoretically. QCD's phenomenology may then serve as a guide to suggest, identify and understand non-perturbative effects in GR.

In fact, the similarity between QCD and GR field Lagrangians may explain intriguing parallels between on the one hand observations interpreted with dark matter and on the other hand the phenomenology of the strong interaction. For examples, the total masses of galaxies and galaxy clusters are much larger than the sum of the masses of their known components, just like for hadrons; galaxies and hadrons share similar mass-rotation correlations (the Tully-Fisher relation and Regge trajectories, respectively); the large-scale balancing of gravitational attraction by dark energy - known as the cosmic coincidence problem - may be paralleled with the large-distance suppression of the strong interaction into a much weaker Yukawa force; the universe's large scale stringy structure observed by weak gravitational lensing is evocative of massive bodies, such as galaxies - or at larger scale two galaxy clusters - linked by a QCD-like string/flux tube.

These parallels and the similar structure of GR and QCD's Lagrangians suggest that massive galactic objects such as galaxies or cluster of galaxies have triggered the non-perturbative regime of GR and that dark matter and dark energy are manifestations of it. It is clear that the selfinteractions terms in GR have the right effects to explain dark matter and dark energy, the question being whether galaxies are massive enough to trigger these effects. This is the question we addressed in the second part of this paper. In QCD, the non-perturbative regime arises for distances greater than $2 \times 10^{-16} \mathrm{~m}$, while we find that for GR it arises at galactic scales. The non-perturbative effects then explain quantitatively the dynamics of spiral galaxies and clusters [37]. In particular, in the case spiral galaxies, the logarithmic potential resulting from the strong field non-linearities trivially yields flat rotation curves. Finally, for a uniform and homogeneous spherical distribution of matter, the non-linearity effects should balance out [37]. Evidences for the consequent correlation expected between galactic ellipticity and galactic dark mass have been found [6]. We thus have a natural explanation for dark matter and dark energy, which according to the method developed and tested in this article, agrees with galactic and cluster dynamics. With the most probable phasespaces for WIMPS and axions dark matter candidates now ruled out, it is important to consider the present explanation for the nature of Dark Matter and Dark Energy.

Acknowledgements The author thanks S. J. Brodsky, C. MunozCamacho, F. X. Girod-Gard, P. Hoyer, J.-F. Mathiot, A. Sandorfi, S. Širca, B. Terzić and X. Zheng for useful discussions on the work reported here. 
Open Access This article is distributed under the terms of the Creative Commons Attribution 4.0 International License (http://creativecomm ons.org/licenses/by/4.0/), which permits unrestricted use, distribution, and reproduction in any medium, provided you give appropriate credit to the original author(s) and the source, provide a link to the Creative Commons license, and indicate if changes were made.

Funded by SCOAP ${ }^{3}$.

\section{A Method}

To numerically compute $G_{2 p}\left(x_{1}-x_{2}\right)$, Eq. (4), a cubic lattice of $N^{3}$ sites is used. Each site is associated with a field value $\phi$. The set of $N^{3}$ values is called a field configuration. These values should be such that they minimize $S$, i.e. the field obeys the Euler-Lagrange equations of motion. To determine these values, a Wick rotation is first performed so that $\mathrm{e}^{-\mathrm{i} S} \rightarrow \mathrm{e}^{-S}$. The action $S$ is unchanged since in the static case, the Euclidean and Minkowski actions are the same. One then proceeds iteratively, following the Metropolis algorithm: for each of the $N^{3}$ sites, $S$ is computed. Next, the value of $\phi$ at a site is shifted randomly and the change in action $\Delta S$ is computed. If $\Delta \leq 0$, the change tends to minimize the action. In this case, the new $\phi$ value is retained because it makes the field configuration closer to what it must be in reality. If $\Delta S>0$, the new $\phi$ value is kept if $\mathrm{e}^{-\Delta S}>\zeta$, with $\zeta$ a random number between 0 and 1 , and rejected otherwise. While this operation is being applied to all the sites, the new configuration of $\phi$ converges toward the configuration obeying the equations of motion. That is, the probability distribution of the field configuration follows $\mathrm{e}^{-S}$. The procedure is repeated enough times and the results are averaged until they converge to the physical solution, while the uncertainty stemming from the random method is minimized. We remark that with this method $Z \equiv 1$ in Eq. (4).

\section{B Practical example of a numerical simulation}

We provide here a practical example of static potential computation for the $\left(g \phi \partial_{\mu} \phi \partial^{\mu} \phi+g^{2} \phi^{2} \partial_{\mu} \phi \partial^{\mu} \phi\right)$ theory. The $g^{2} \phi^{4}$ case is simpler and follows the same method.

\section{B.1 First simulation}

1. The first step is to choose an initial configuration for $\phi$, that is to choose $N^{3}$ values of $\phi$, one for each of the $N^{3}$ lattice sites. The $\phi$ can be set to 0 or to random values $-\varepsilon<\phi<+\varepsilon$. The optimal value for $\varepsilon$, about 1 , will be discussed in the next step. For a simple first simulation, the values of $\phi$ at the boundaries can be set to 0 (see Appendix $\mathrm{C}$ for a discussion). A small $N$ value, e.g. $N=$ 11 , can be used initially (it can be increased once the program is debugged and optimized).

2. The initial configuration is arbitrary. Before spending CPU time to compute $G_{2 p}\left(x_{1}-x_{2}\right)$, one must "thermal- ize" the configuration, i.e. modify it so that it is already close to the physical configuration. To do this, the configuration is updated $10 \times N_{\text {cor }}$ times (take $N_{\text {cor }}=20$ ) except at the boundaries where $\phi$ stays 0 . Each update follows the Metropolis procedure discussed in Sect. A. For each update of the configuration, the $(N-2)^{3}$ $\phi$ are offset by random numbers distributed uniformly between $\pm \varepsilon$. For debugging ease at this stage, the lattice version of the action; see e.g. Eq. (17) for the $\left(g \phi \partial_{\mu} \phi \partial^{\mu} \phi+g^{2} \phi^{2} \partial_{\mu} \phi \partial^{\mu} \phi\right)$ theory, should be used. The configuration updates can subsequently be sped up considerably by using only the local change in the action; see Eq. (18). Once Eq. (18) is used, $N$ can be increased to 25 .

3. To further speed-up the simulation and minimize configuration correlations, $\varepsilon$ must be optimized. The optimal value for $\varepsilon$ is such that during updates, about half of the $\phi$ values are changed once the configuration is thermalized.

4. One can now compute $G_{2 p}\left(x_{1}-x_{2}\right)$. For simplicity, we can choose $x_{1}=(0,0,0)$, i.e. $x_{1}$ is the center of the lattice, choose $x_{2}$ along a single direction, say $x$, and stop just before the boundary. In that case, $G_{2 p}( \pm n)=$ $\phi(0,0,0) \phi( \pm n a, 0,0)$, with $1<n<(N-3) / 2$, the number of possible distances. The computations are repeated $N_{\mathrm{S}}$ times and averaged for each given distance $n$. Use $N_{\mathrm{s}}=5000$. To suppress correlations, between each computation of $G_{2 p}$, the configuration is updated $N_{\text {cor }}$ times following step 2. (This is necessary because two configurations differing by only one update are still similar, hence correlating the two $G_{2 p}$ and biasing the statistical averaging.)

For a simulation using Eq. (18), with $N^{3}=(25)^{3}$, $N_{\text {cor }}=20, N_{\mathrm{s}}=5000$ and $\varepsilon=1.2$, a typical simulation takes approximately $3 \mathrm{~min}$ on a MacBook Pro equipped with a $2.8 \mathrm{GHz}$ Intel Core 2 Duo processor. To assess how this compares to other numerical approaches one can extend this figure to state-of-art, 10 pflops, lattice machines. We can also take advantage of the fact that, once the boundary conditions discussed in Appendix C are implemented, the Green function can be computed in any possible direction at essentially no extra CPU-cost. In that case the relative precision of the calculation would be $2 \times 10^{-7}$ for a 1-min calculation, comparable to the precision of a 32-bit machine.

\section{B.2 Refinements}

\section{B.2.1 Faster updating}

1. To update a configuration, the discretized expression of the action is needed. The action below is the same as 
Eq. (12) but ignoring term of order $g^{3}$ or higher. Also, a field mass $m$ is added for checking purposes. There is one source located at $x_{1}$ :

$$
\begin{aligned}
S= & \frac{1}{2} \int \mathrm{d}^{3} \phi\left[\left(\partial_{i} \phi \partial^{i} \phi+g \phi^{2} \partial_{i} \phi \partial^{i} \phi\right.\right. \\
& \left.\left.+g^{2} \phi \partial_{i} \phi \partial^{i} \phi-g \phi \delta\left(x_{1}\right)+m^{2} \phi^{2}\right)\right] .
\end{aligned}
$$

The $\phi^{n} \partial \phi \partial \phi$ terms can be transformed into $\frac{1}{n+1} \phi^{n+1} \partial^{2} \phi$ by integrating by parts. Recalling that, numerically, $\partial^{2} \phi(x) \approx[\phi(x+a)-2 \phi(x)+\phi(x-a)] / a^{2}$, with $a$ the distance between adjacent nodes, the numerical version of the continuous action, Eq. (16) is

$$
\begin{aligned}
& S=-\frac{1}{2} \sum_{n_{x}=2, n_{y}=2, n_{z}=2}^{N-1, N-1, N-1}\left(\left[\phi\left(n_{x}+1, n_{y}, n_{z}\right)\right.\right. \\
& +\phi\left(n_{x}-1, n_{y}, n_{z}\right)+\phi\left(n_{x}, n_{y}+1, n_{z}\right) \\
& +\phi\left(n_{x}, n_{y}-1, n_{z}\right) \\
& +\phi\left(n_{x}, n_{y}, n_{z}+1\right)+\phi\left(n_{x}, n_{y}, n_{z}-1\right) \\
& \left.-6 \phi\left(n_{x}, n_{y}, n_{z}\right)\right] \\
& \times\left[\phi\left(n_{x}, n_{y}, n_{z}\right)+g \phi^{2}\left(n_{x}, n_{y}, n_{z}\right)+g^{2} \phi^{3}\left(n_{x}, n_{y}, n_{z}\right)\right] \\
& \left.+g \phi\left(n_{x}, n_{y}, n_{z}\right) \delta_{x_{1}, y_{1}, z_{1}}-m^{2} \phi^{2}\left(n_{x}, n_{y}, n_{z}\right)\right),
\end{aligned}
$$

where $n_{x}, n_{y}$ and $n_{z}$ are the site indices in the $x, y$ and $z$ directions, respectively, and the distance between consecutive nodes has been set to $a=1$ (in lattice units).

2. Using Eq. (17) directly makes the simulation computationally inefficient. However, since only the difference between the actions before and after an update is used, we do not need to sum the action over all the sites: only local terms near the site $\left(n_{x}, n_{y}, n_{z}\right)$ that do not cancel in the difference are important. The difference of the actions is

$$
\begin{aligned}
& S_{\text {new }}-S_{\text {old }} \equiv \Delta S=-\frac{1}{2}\left(\left[\phi_{\text {old }}\left(n_{x}+1, n_{y}, n_{z}\right)\right.\right. \\
& \quad+\phi_{\text {old }}\left(n_{x}, n_{y}+1, n_{z}\right)+\phi_{\text {old }}\left(n_{x}, n_{y}, n_{z}+1\right) \\
& \quad-6 \phi_{\text {new }}\left(n_{x}, n_{y}, n_{z}\right) \\
& \quad+\phi_{\text {old }}\left(n_{x}-1, n_{y}, n_{z}\right)+\phi_{\text {old }}\left(n_{x}, n_{y}-1, n_{z}\right) \\
& \left.\quad+\phi_{\text {old }}\left(n_{x}, n_{y}, n_{z}-1\right)\right] \\
& \quad \times\left[\phi_{\text {new }}\left(n_{x}, n_{y}, n_{z}\right)+g \phi_{\text {new }}^{2}\left(n_{x}, n_{y}, n_{z}\right)\right. \\
& \left.\quad+g^{2} \phi_{\text {new }}^{3}\left(n_{x}, n_{y}, n_{z}\right)\right] \\
& \quad-\left[\phi_{\text {old }}\left(n_{x}+1, n_{y}, n_{z}\right)+\phi_{\text {old }}\left(n_{x}, n_{y}+1, n_{z}\right)\right. \\
& \quad+\phi_{\text {old }}\left(n_{x}, n_{y}, n_{z}+1\right) \\
& \quad-6 \phi_{\text {old }}\left(n_{x}, n_{y}, n_{z}\right)+\phi_{\text {old }}\left(n_{x}-1, n_{y}, n_{z}\right) \\
& \quad+\phi_{\text {old }}\left(n_{x}, n_{y}-1, n_{z}\right) \\
& \left.\quad+\phi_{\text {old }}\left(n_{x}, n_{y}, n_{z}-1\right)\right]\left[\phi_{\text {old }}\left(n_{x}, n_{y}, n_{z}\right)\right. \\
& \quad+g \phi_{\text {old }}^{2}\left(n_{x}, n_{y}, n_{z}\right)
\end{aligned}
$$

$$
\begin{aligned}
& \left.+g^{2} \phi_{\text {old }}^{3}\left(n_{x}, n_{y}, n_{z}\right)\right]+\left[\phi_{\text {new }}\left(n_{x}, n_{y}, n_{z}\right)\right. \\
& \left.-\phi_{\text {old }}\left(n_{x}, n_{y}, n_{z}\right)\right] \\
& \times\left[\phi_{\text {old }}\left(n_{x}+1, n_{y}, n_{z}\right)+\phi_{\text {old }}\left(n_{x}, n_{y}+1, n_{z}\right)\right. \\
& +\phi_{\text {old }}\left(n_{x}, n_{y}, n_{z}+1\right) \\
& +\phi_{\text {old }}\left(n_{x}-1, n_{y}, n_{z}\right)+\phi_{\text {old }}\left(n_{x}, n_{y}-1, n_{z}\right) \\
& +\phi_{\text {old }}\left(n_{x}, n_{y}, n_{z}-1\right) \\
& +g\left[\phi_{\text {old }}^{2}\left(n_{x}+1, n_{y}, n_{z}\right)+\phi_{\text {old }}^{2}\left(n_{x}, n_{y}+1, n_{z}\right)\right. \\
& +\phi_{\text {old }}^{2}\left(n_{x}, n_{y}, n_{z}+1\right)+\phi_{\text {old }}^{2}\left(n_{x}-1, n_{y}, n_{z}\right) \\
& +\phi_{\text {old }}^{2}\left(n_{x}, n_{y}-1, n_{z}\right) \\
& \left.+\phi_{\text {old }}^{2}\left(n_{x}, n_{y}, n_{z}-1\right)\right]+g^{2}\left[\phi_{\text {old }}^{3}\left(n_{x}+1, n_{y}, n_{z}\right)\right. \\
& +\phi_{\text {old }}^{3}\left(n_{x}, n_{y}+1, n_{z}\right)+\phi_{\text {old }}^{3}\left(n_{x}, n_{y}, n_{z}+1\right) \\
& +\phi_{\text {old }}^{3}\left(n_{x}-1, n_{y}, n_{z}\right) \\
& \left.+\phi_{\text {old }}^{3}\left(n_{x}, n_{y}-1, n_{z}\right)+\phi_{\text {old }}^{3}\left(n_{x}, n_{y}, n_{z}-1\right)\right] \\
& +g\left[\phi_{\text {new }}\left(n_{x}, n_{y}, n_{z}\right)-\phi_{\text {old }}\left(n_{x}, n_{y}, n_{z}\right)\right] \delta_{x_{1}, y_{1}, z_{1}} \\
& \left.-m^{2}\left[\phi_{\text {new }}^{2}\left(n_{x}, n_{y}, n_{z}\right)-\phi_{\text {old }}^{2}\left(n_{x}, n_{y}, n_{z}\right)\right]\right)
\end{aligned}
$$

where $\phi_{\text {old }}$ and $\phi_{\text {new }}$ denote the fields before and after update, respectively.

\section{B.2.2 Statistics increase}

To gain statistics at low CPU cost, $G_{2 p}$ can be computed along the $\mathrm{y}$ and $\mathrm{z}$ axes as well. To further gain statistics, it can also be computed in more directions than just the three orthogonal axes. However, in that case, boundary conditions $\phi=0$ on a sphere of radius $N / 2$ must be imposed. Otherwise, with the cubic zero boundary, any strong $\phi$ autocorrelation would suppress $G_{2 p}$ calculated along one of the axis compared to a $G_{2 p}$ calculated along e.g. a diagonal.

\section{Boundary conditions}

The two-point Green function $G_{2 p}(x)$ quantifies the field autocorrelation. Weak correlations imply that the field values in most of the lattice volume are independent of their values at the boundary. In that case the choice of boundary conditions is not critical: see Fig. 9 where $G_{2 p}$ is calculated for a two-source system with free-fields. One can use periodic boundary conditions, Dirichlet boundary conditions ( $\phi=0$ at the boundaries), or random values within the range $-\varepsilon<\phi<+\varepsilon$ ( $\varepsilon$ is the optimal range within which $\phi$ is randomly shifted; see Appendix B.1). Likewise, in Fig. $2 G_{2 p}$ is computed for different boundary conditions in the $g^{2} \phi^{4}$ case, using the Lagrangian in Eq. (1). The quartic term generates an effective mass. Thus the field correlation length is shortened and the choice of boundary condition is even less important. 


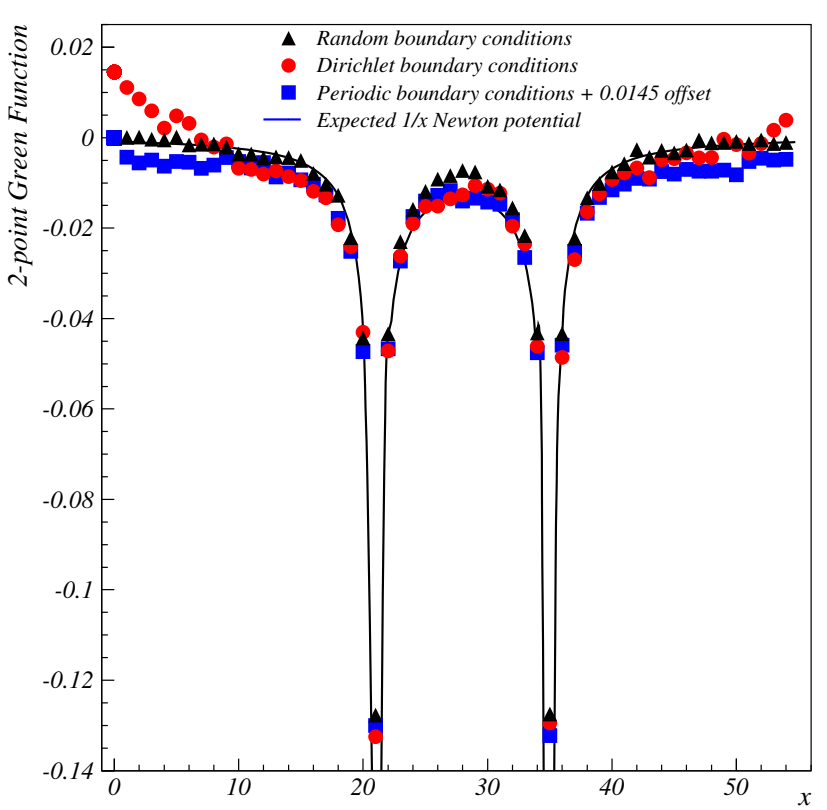

Fig. 9 Independence of the simulation on the choice of boundary conditions. The results are obtained when keeping only $\partial \phi \partial \phi$ and the matter terms in the Lagrangian ( $1 / x$ free massless field case). The potential $G_{2 p}$ is plotted as a function of distance. The simulations were run for a lattice size $N=55$, a coupling value $g=10^{-2}$, a correlation coefficient (see Sect. B.1) $N_{\text {cor }}=20$ and for $N_{\mathrm{s}}=3.5 \times 10^{4}$ configurations. Two sources are located on the $x$-axis at $d= \pm 7$ from the lattice center at $x_{\text {center }}=28$. The circles are for Dirichlet boundary conditions. The squares are obtained with periodic boundary conditions. The triangles are for random field boundary conditions. Except near the boundaries and except for a constant offset of 0.0145 added to the result obtained with the Dirichlet conditions (irrelevant since $G_{2 p}$ is the potential), all results agree and display the expected $1 / x$ potential for each sources (continuous curve)

We remark that, although Eq. (1) contains a non-linear term, i.e. a term involving field self-interactions, the total potential is well represented by the sum of the two individual potentials, thus obeying the field superposition principle. This is because there is no cubic term due to the equation of motion, and because the quartic term essentially acts as a mass term.

In contrast, strong field autocorrelations imply that the field at any position on the lattice could strongly depend on the boundary values. The correlation length becomes an additional relevant physical scale to the problem. This is the case with the $\left(g \phi \partial_{\mu} \phi \partial^{\mu} \phi+g^{2} \phi^{2} \partial_{\mu} \phi \partial^{\mu} \phi\right)$ theory, where covariant derivatives are present in each of the Lagrangian terms and thus increase the field autocorrelation when $g$ becomes large. If the correlation length is sufficiently large and becomes comparable to the lattice size then $\phi$ on any lattice site will depend on the choice of the boundary conditions. In that case, $G_{2 p}$ acquires an unphysical dependence on the lattice size $N$, or equivalently on the lattice spacing for a given system size. This unphysical phenomenon can be suppressed by using large lattices made of an inner volume where the full action is used, and an outer volume where we

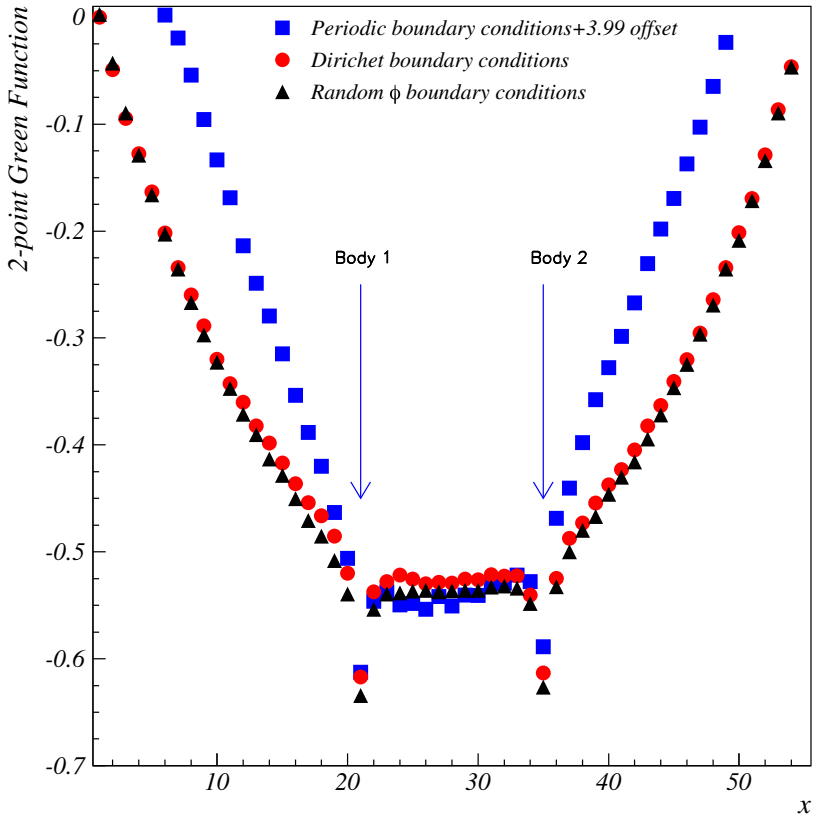

Fig. 10 Same as Fig. 9 but using the full $\left(g \phi \partial_{\mu} \phi \partial^{\mu} \phi+g^{2} \phi^{2} \partial_{\mu} \phi \partial^{\mu} \phi\right)$ theory Lagrangian, Eq. (12). The results are essentially the same for Dirichlet and random field boundary conditions. They are qualitatively similar for periodic boundary conditions but quantitatively, the force between the two sources differs by up to a factor of two. The cause of this difference originates from spurious effects in the periodic boundary case due to the long correlation length allied with the infinite repetitions of the sources

set $g=0$ (free-field case). The outer region provides a transition buffer between the system and the arbitrary $\phi$-values at the boundaries. This suppresses the dependence on the choice of the field at the boundary and the $N$-dependence; see Figs. 10 and 13 where the Lagrangian of Eq. (12) was used. The results using the random and Dirichlet boundary conditions agree well. The results with periodic boundary condition are similar qualitatively but differ quantitatively. The force computed under these conditions is about a factor of two larger than with the random and Dirichlet boundary conditions. It is expected that for correlation lengths comparable to or larger than the lattice size, the repeated lattice pattern of the image sources spuriously influences the field autocorrelation function anywhere in the lattice, which is likely the cause for this difference between the result using the periodic boundary conditions and the results using the two other conditions. A similar effect is discussed in the context of linear potential in Refs. [15,37].

Another example of field with long correlation length is discussed in Appendix D.1.2 where we recover the known $1 / x$ free massless field potential in two-dimensional space. This logarithmic potential represents fields that are significantly autocorrelated and exemplifies the need for a careful handling of boundary conditions to avoid spurious boundary effects. 
Fig. 11 Potentials obtained for the free (massless and massive) field cases (left). The free massless field potential is shown by the squares $(m=0)$. The free massive field (Yukawa) potentials are obtained for different values of the field mass, $m=0.1$ (up triangles), $m=0.2,($ down triangles $)$ and $m=0.4$ (open circles). Residuals from the $\mathrm{e}^{-m x} / x$ expectation are shown on the right

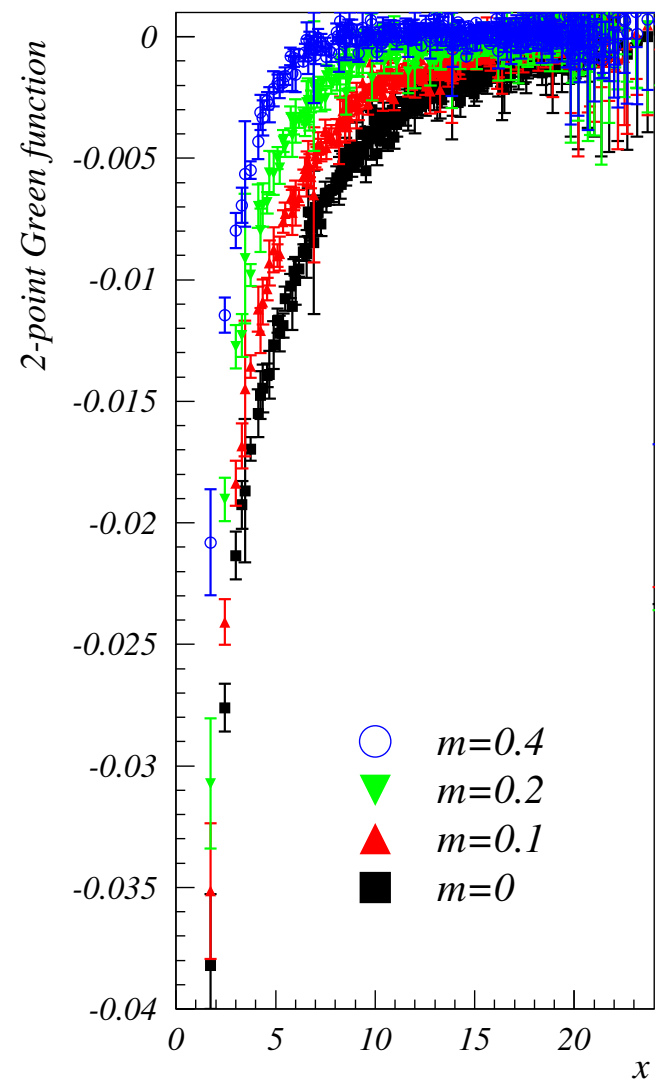

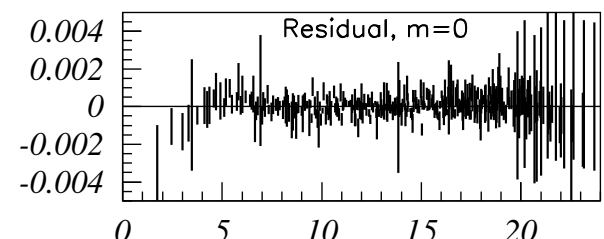
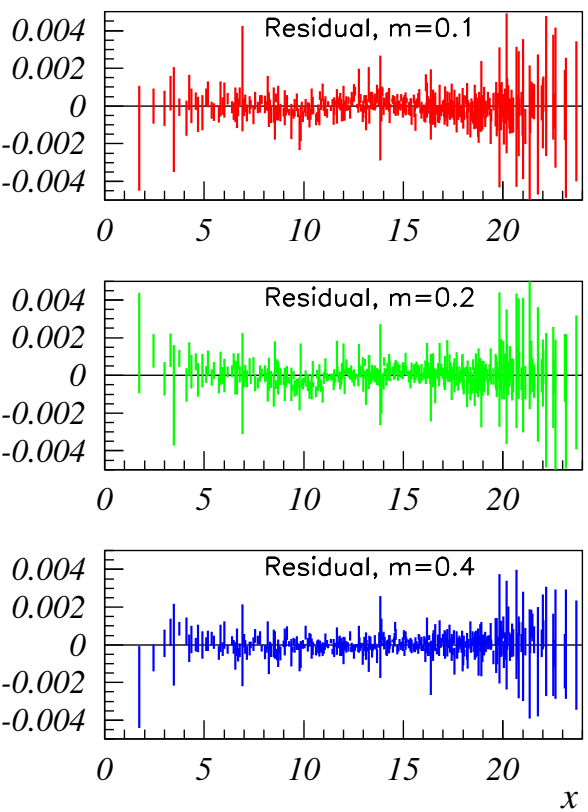

\section{Method validation}

D.1 Recovering analytically known potentials

The method can be validated by verifying that analytically known potentials are recovered. In this section we compute the free massless field, free massive field (Yukawa) and $g^{2} \phi^{4}$ theory static potentials in the case of three dimensions. We also compute the massless and massive free-field static potentials in two-dimensional space. We then compare them to their expected analytical forms.

\section{D.1.1 Potentials in three-dimensional space}

In the massless free-field case, one should recover a $G_{2 p}(x)$ $\propto 1 / x$ potential. Furthermore, if one adds to the Lagrangian another quadratic term corresponding to a field mass term, $m^{2} \phi^{2}$, one should recover a Yukawa potential, $G_{2 p}(x) \propto$ $\mathrm{e}^{-m x} / x$. Such simulations were ran using a lattice size $N=$ $36, N_{\mathrm{s}}=10^{4}$ configurations, a decorrelation coefficient (see Sect. B.1) $N_{\text {cor }}=10$ and random field boundary conditions. The results are shown in Fig. 11. The simulation agrees well the expected potentials in both the free massless field and the Yukawa cases.

As discussed in the main text in Sect. 4.1 the $g^{2} \phi^{4}$ case is analytically known [8]. We recovered potentials well fit by a Yukawa potential or by the convolution of the Jacobi elliptic function $\operatorname{sn}(x,-1)$, the expected function describing $\phi$ [8]. The effective field mass stemming from $g^{2} \phi^{4}$, shown in function of $Q^{2}$ in Fig. 7 qualitatively agrees with typical QCD expectation. Injecting the running field mass in the Yukawa potential form and Fourier transforming to position space yield the static potential shown in Fig. 8. It displays a linear behavior in the $x$-range relevant to hadron physics and agrees well with the Cornell potential. In all, the $g(x)^{2} \phi^{4}$ theory recovers the principal confinement features expected from strong interaction. If $g^{2} \phi^{4}$ is the classical limit of QCD as proposed in [18], the good match between the $g(x)^{2} \phi^{4}$ theory and Cornell potentials suggests that the missing quantum effects can be fully encapsulated in the running of the coupling and that the method discussed here does provide relevant approximations to QCD and GR.

\section{D.1.2 Potentials in two-dimensional space}

We verify here that in two-dimensional space, a logarithmic potential is recovered in the case of $g=0$ (now integrating the Lagrangian density only over two dimensions). The simulation was performed with $N=45, N_{\text {cor }}=15$ and $N_{\mathrm{s}}=2.5 \times 10^{4}$. We checked the dependence on field values at the boundaries by performing the calculation for four different cases: 


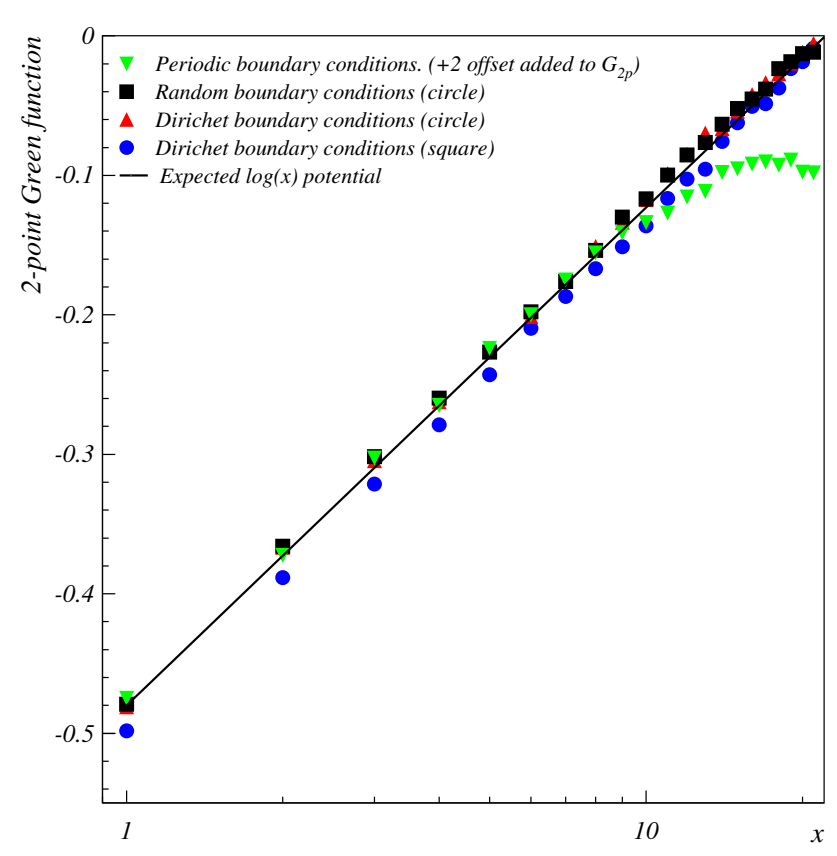

Fig. 12 Potentials in a two-dimensional space obtained when keeping only the $\partial \phi \partial \phi$ term in the Lagrangian. In this semi-log graph, the expected logarithmic potential is rectilinear. The various symbols represent different boundary conditions; see main text

1. Dirichlet boundary conditions on the edges of the square lattice.

2. Dirichlet boundary conditions outside a circle of diameter $N$ centered on the lattice center at $x_{\text {center }}=y_{\text {center }}=$ $N / 2$.

3. Random field boundary conditions outside a circle of diameter $N$ centered on the lattice center.

4. Periodic boundary conditions on the square lattice.

As expected, we obtain $G_{2 p}(x) \propto \log (x)$; see Fig. 12. The agreement breaks down at a distance of about 10 lattice units from the boundary in the case of periodic boundary conditions. This is because for indices $(i, j), \phi(x=i, y=j)$ is the same as $\phi(x=i+N, y=j+N)$, i.e. they are fully correlated. Consequently, $G_{2 p}$ is periodic with a period $N$. This effect is not seen in three dimensions where the correlation quickly falls as $1 / x$. The effect is important for two dimensions where the correlation falls more slowly, as $\log (x)$. This underlines the importance of choosing the boundary conditions with care, so that the simulation result is as independent as possible of the field values at the boundaries. Clearly, simple periodic boundary conditions (i.e. without the buffer region discussed in Appendix C) are ill-suited in the case of strongly autocorrelated fields, such as in the present twodimensional case and, a fortiori, the three-dimensional case with large field self-interaction terms.

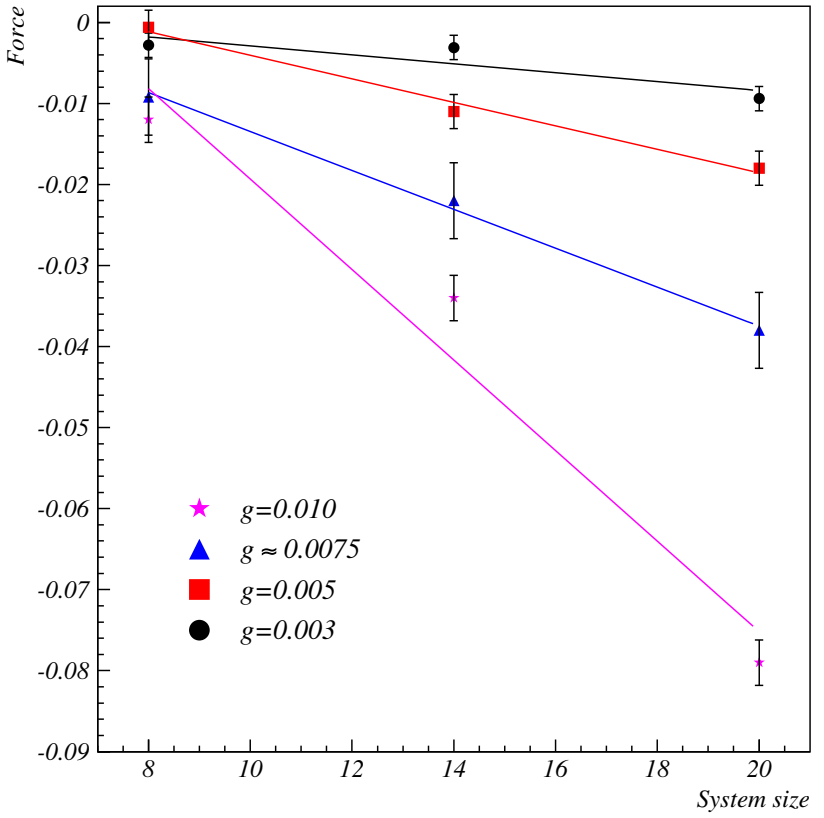

Fig. 13 Dependence of the force between two sources with the distance $L=2 d$ separating them (system size). The four different symbols are results for different values of the coupling $g$. (Details of these calculations are discussed in Sect. E.1.3.) Points are fit with a linear function, yielding an overall $\chi^{2} / n d f$ of 1.4. The uncertainties are taken as the difference between the force extracted near source 1 and the force near source 2

\section{E Dependence on lattice parameters}

The lattice parameters $N$ (lattice size), $N_{\mathrm{s}}$ (statistics), $N_{\text {cor }}$ (decorrelation parameter; see Appendix B.1) and the lattice spacing $a$ (distance between adjacent nodes) do not correspond to any physical property of a system. Results should consequently be independent of them. We verify this in this appendix, first for the quadratic case (massless and massive free-fields) and then for the $g^{2} \phi^{4}$ and $\left(g \phi \partial_{\mu} \phi \partial^{\mu} \phi+g^{2} \phi^{2} \partial_{\mu} \phi \partial^{\mu} \phi\right)$ cases.

\section{E.1 Dependence on the system size}

A long correlation length implies long-range effects. If the correlation length is similar to the system size, these long range effects should depend on the physical size of the system, or equivalently on the distance at which the field becomes negligible, since these two scales must be related. See Ref. [15] for an example of such a phenomenon in an analytical calculation context. Our calculations for the $\left(g \phi \partial_{\mu} \phi \partial^{\mu} \phi+g^{2} \phi^{2} \partial_{\mu} \phi \partial^{\mu} \phi\right)$ theory, Eq. (12), do show that the larger the system, the stronger the long-range effects. This can be seen in Fig. 13 where the force between two sources separated by a distance $2 d$ is plotted versus $2 d$ for the same lattice unit $a$. The dependence of the force on $2 d$ is linear, as expected. 
Fig. 14 Potentials for various values of parameters used in the simulation. Only the quadratic term $\partial \phi \partial \phi$ in the Lagrangian (massless free-field case) was kept. For these simulations the system size $N$, the decorrelation parameter $N_{\text {cor }}$, and the statistics $N_{\mathrm{s}}$, are varied. In the bottom right panel, the residuals between the results and the expected $1 / x$ behavior are shown. The vertical axis span is the same as for the three first panels and magnified by a factor 3 for the bottom right panel showing the residuals
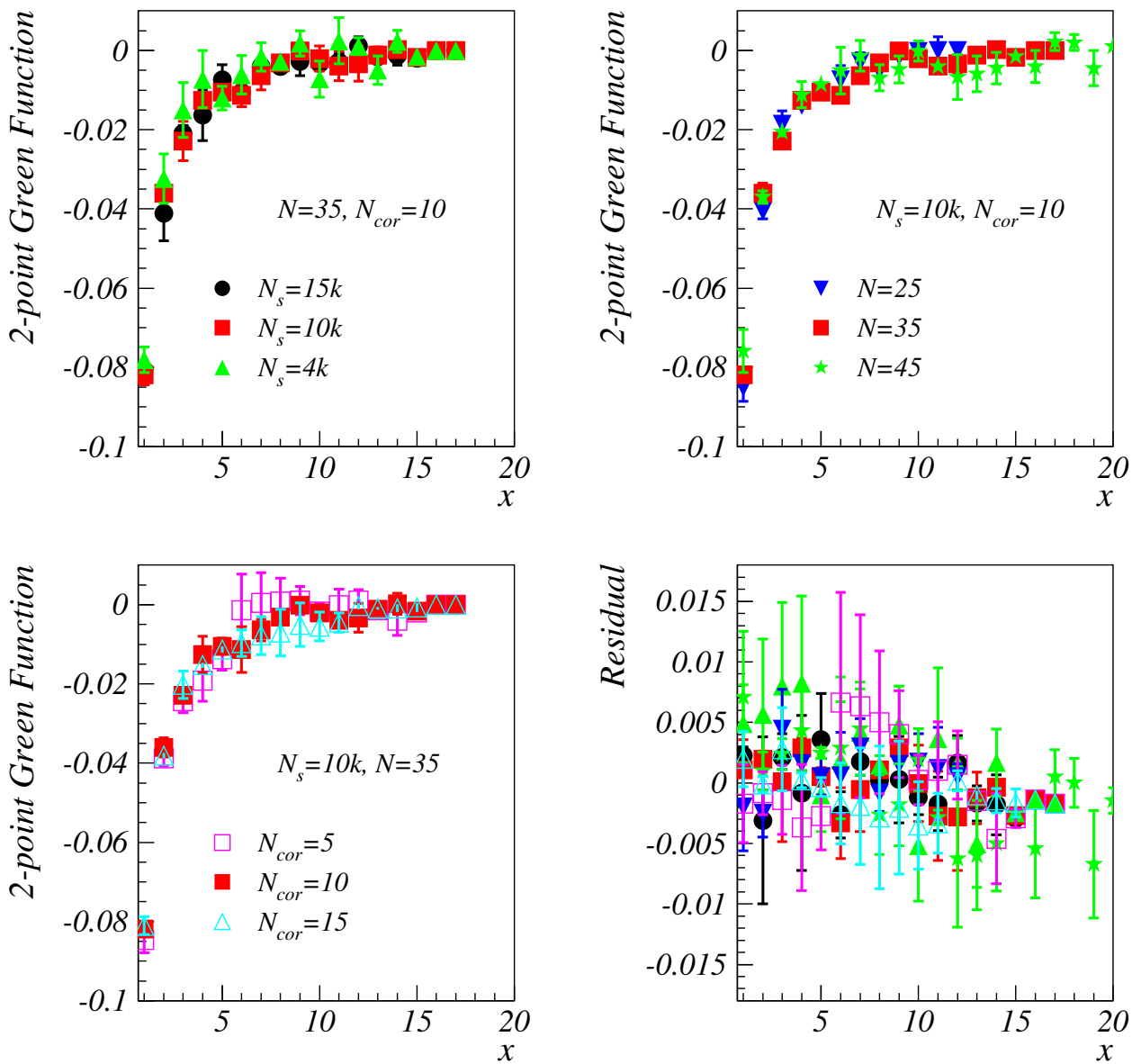

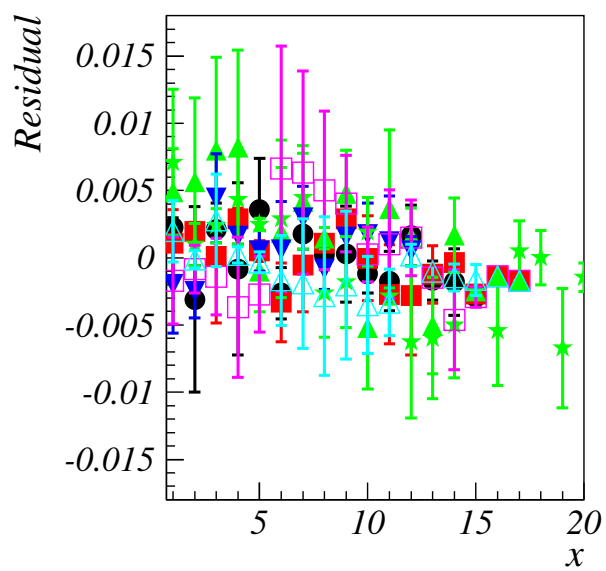

\section{E.1.1 Free massless and massive field cases}

We verified in the free massless and massive field cases the independence of $G_{2 p}$ on $N, N_{\text {cor }}$ and $N_{\mathrm{s}}$; see Fig. 14. In the top left plot, the effect of statistics $\left(N_{\mathrm{S}}\right)$ is checked. In the top right plot, the effect of the lattice size $(N)$ is checked. In the bottom left plot, possible correlations between configurations $\left(N_{\text {cor }}\right)$ are checked. In the bottom right plot, the residuals between these results and the expected $1 / x$ behavior are shown. It appears that $N_{\text {cor }}=10$ is adequate for a simulation in the quadratic case.

The results must also be independent of the physical value of the lattice spacing unit $a$, i.e. calculations on lattices of different sizes $N$ but corresponding to the same physical size must yield the same result. The values of $G_{2 p}$ obtained with the simulation performed for different values of $N$ are plotted on top of each other in Fig. 15. Their respective $x$ dependencies are scaled by a factor $L / N$, where $L$ is the system physical size, taken to be 100 in Fig. 15. The dimen-

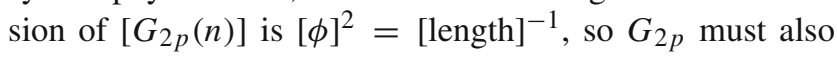
be scaled by a factor $N / L$. Finally, if a mass term is present (Yukawa case), it must be scaled in the simulation by a factor $N / L$, since $m$ scales as [length] ${ }^{-1}$. The left panel in Fig. 15 shows the massless free-field case and the right panel the
Yukawa case. In both instances, the calculations for different $N$ values agree well. We next verify the independence from the lattice parameters, first in the $g^{2} \phi^{4}$ theory and then in the case of $\left(g \phi \partial_{\mu} \phi \partial^{\mu} \phi+g^{2} \phi^{2} \partial_{\mu} \phi \partial^{\mu} \phi\right)$ theory.

\section{E.1.2 $g^{2} \phi^{4}$ case}

The dependence of the results with variation of the lattice size $N$, decorrelation parameter $N_{\text {cor }}$, and statistics $N_{\mathrm{s}}$ is shown in Fig. 16. We used $g^{2}=0.5$, which yields an effective Yukawa behavior characterized by an effective field mass $m_{\text {eff }} \simeq 0.72$. There is an excellent agreement between the data indicating no dependence on the choice of lattice parameters.

\section{E.1.3 $\left(g \phi \partial_{\mu} \phi \partial^{\mu} \phi+g^{2} \phi^{2} \partial_{\mu} \phi \partial^{\mu} \phi\right)$ theory case}

Dependence on lattice size. In Fig. 17, we show $G_{2 p}(x)$ computed for different lattice sizes varying from $N=45$ to $N=95$. Dirichlet boundary conditions were used. The distance between the two sources is $2 d=14$ lattice spacings for all $N$. For ease of comparison, arbitrary constants were added to the $G_{2 p}(x)$ s so that the troughs at distance $x=21$ in the top panel overlap. (Constants do not affect the forces 

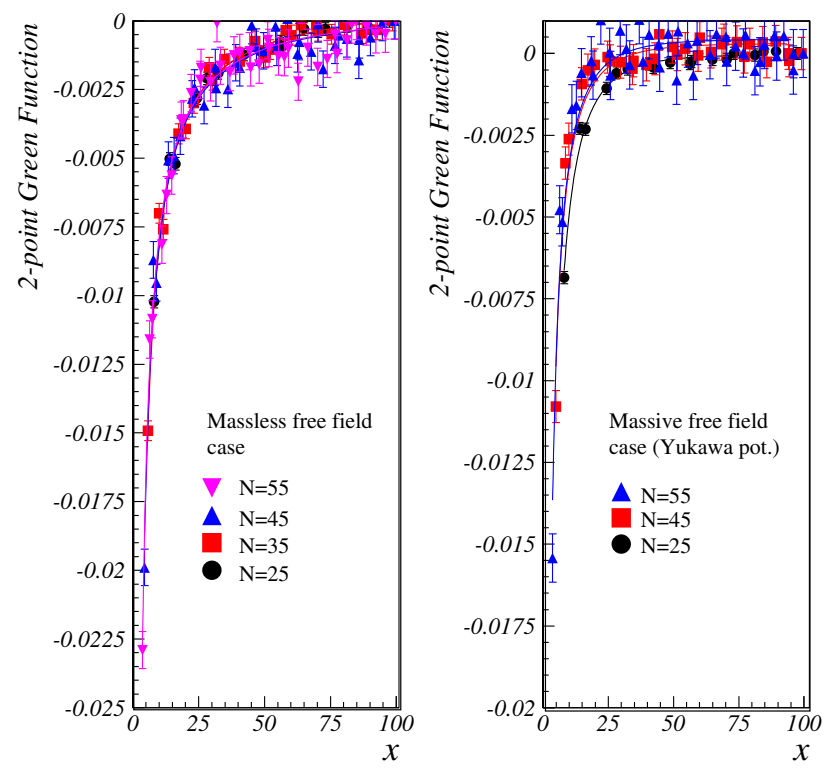

Fig. 15 Verification that $G_{2 p}$ calculated numerically is invariant with respect to changing the lattice spacing units. In the left panel, the massless free-field case $(\mathcal{L}=\partial \phi \partial \phi)$ is shown. The case for $\mathcal{L}=$ $\partial \phi \partial \phi+m^{2} \phi^{2}$ (Yukawa potential) is shown on the right panel. Statistics are $N_{\mathrm{s}}=5000$ for results with $N=25, N_{\mathrm{s}}=4000$ for massless free-field results with $N=35$ and $N_{\mathrm{s}}=2000$ for the other results. A decorrelation parameter of $N_{\text {cor }}=10$ has been used. A mass $m=0.05$ has been used for the Yukawa potential. The lines are the best fits to the data points using the expected functional forms $1 / x$ and $\mathrm{e}^{-m x} / x$ for the massless and massive cases, respectively. Their color matches the symbol colors
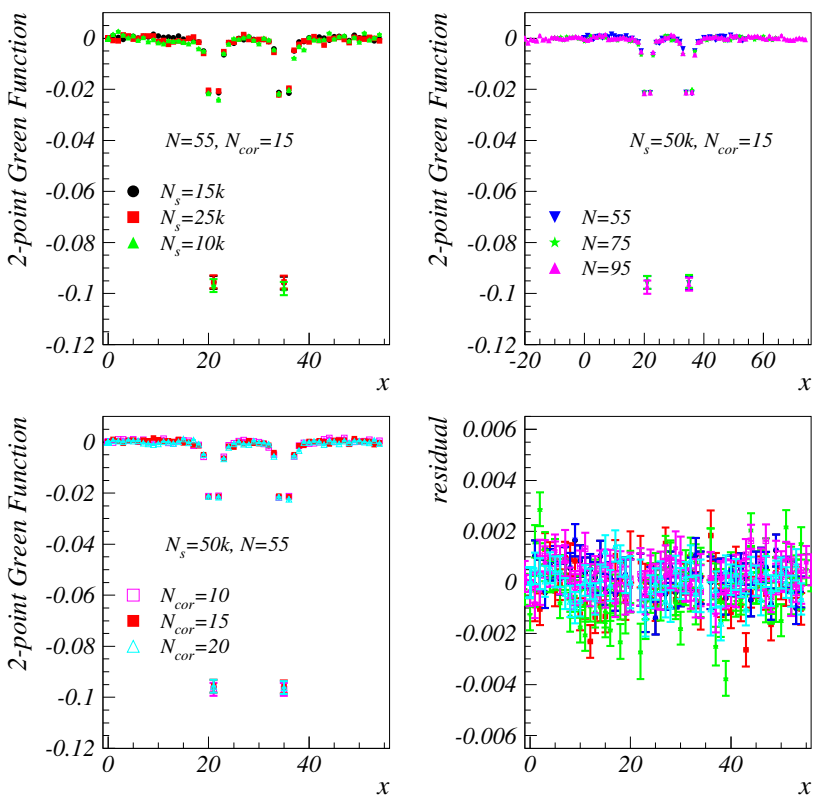

Fig. 16 Potentials for various values of parameters used in the simulation. The $g^{2} \phi^{4}$ Lagrangian, Eq. (1), is used with $g^{2}=0.5$. The system size, $N$, the decorrelation parameter, $N_{\text {cor }}$, and the statistics, $N_{\mathrm{s}}$, are varied. In the bottom right panel, the residuals between the results and the effective Yukawa behavior are shown. The vertical axis span is the same as for the three first panels and magnified by a factor 10 for the bottom right panel showing the residuals

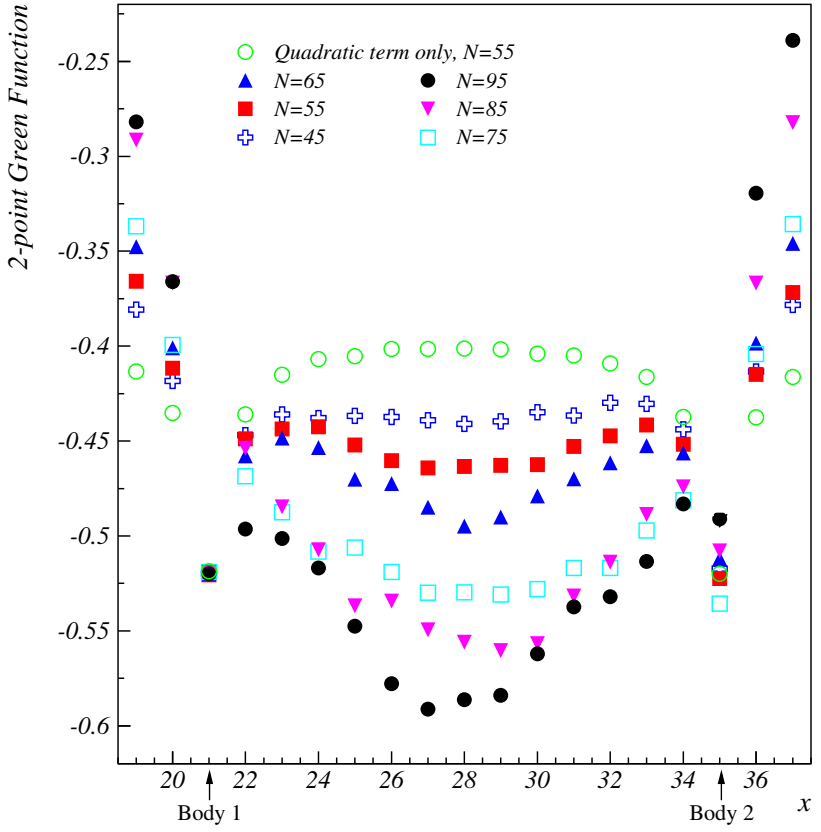

Fig. 17 Potentials computed for different lattice sizes varying from $N=45$ to $N=95$. The Lagrangian in Eq. (12) is used except for the open circles that show the massless free-field case. The two bodies are located on the $x$-axis at $d= \pm 7$ from the lattice center $x_{\text {center }}=28$, $y=0$ and $z=0$. The coupling is $g=7.5 \times 10^{-3}$. The decorrelation parameter is $N_{\text {cor }}=20$ and $N_{\mathrm{s}}=3.5 \times 10^{4}$ paths were used. To facilitate comparing the results, a constant offset is added to the data for each $N$ cases so that the data at the position of the first body, $x=21$, match

resulting from the potentials.) The effect of the cubic and quartic terms on the force is obtained by taking the derivative of $G_{2 p}$ after subtracting the free-field contribution (open circles). There is a $N$-dependence of the force. It is approximately linear with $N$ near the matter locations within the $N$ range checked $(45 \leq N \leq 115)$ for the various cases checked ( $d=4,7$ and 10 , and $\left.3 \times 10^{-3} \leq g \leq 10^{-2}\right)$; see Fig. 18 . This difference in slope between the results for different $N$ is likely a residual effect similar to the physical effect discussed in Sect. E. 1 but associated with the buffer region size or the lattice size rather than the physical size. Consequently, the induced linear $N$-dependence must be corrected for. It can be done, for example, by subtracting the $N$-dependent component. Practically, it is done by taking the $N \rightarrow 0$ (classical) limit.

Dependence on lattice parameters. As shown in Appendix $\mathrm{C}$, the results are mostly independent of the choice of field values at the boundaries. We verify now that the results are also independent of the lattice spacing if $g$ is small enough and the boundaries are sufficiently far from the points where $G_{2 p}$ is calculated. The effect of the cubic term $g \phi \partial_{\mu} \phi \partial^{\mu} \phi$ and quartic term $g^{2} \phi^{2} \partial_{\mu} \phi \partial^{\mu} \phi$ on the force between the two sources, in the classical $N \rightarrow 0$ limit, is shown in Fig. 19. It is given along $x$ by $\mathrm{d}_{2 p}(x) / \mathrm{d} x$ and is 


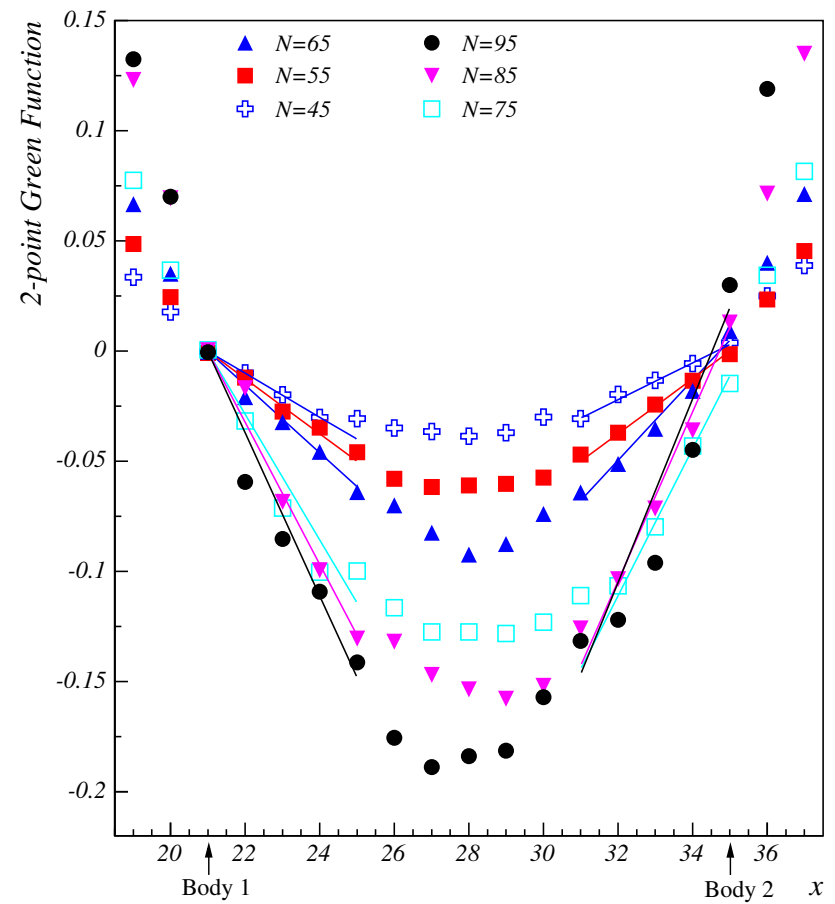

Fig. 18 Same as Fig. 17 but with the free-field potential subtracted. The line segments illustrate the nearly linear trend of the potential

measured a few nodes away from the sources (e.g. around $x=21$ and $x=35$ in Fig. 18). The free massless field contribution $G_{2 p}^{\text {free-field }}(x) \propto\left[1 /\left(x-x_{1}\right)+1 /\left(x-x_{2}\right)\right]$ was first subtracted. (We used the lattice result obtained with $g^{2}=0$ for the subtraction rather than the analytic expression.) The simulation was performed for lattice sizes $45 \leq N \leq 95$, for three different numbers of lattice nodes between the two sources: $2 d=8,14$ and 20, and for couplings $3 \times 10^{-3}<g<10^{-2}$. The correlation parameter was $N_{\text {cor }}=20$, the statistic $N_{\mathrm{s}}=3.5 \times 10^{4}$, and the Dirichlet boundary conditions were used. For the same two-source system, the physical lattice unit $a$ is a factor 7/4 times larger for the $d=4$ calculation compared to the $d=7$ calculation, and a factor $7 / 10$ times smaller for the $d=10$ calculation compared to $d=7$. Since the dimension of $g$ is $\sqrt{[\text { length }]}$ and the force dimension is [length] ${ }^{-2}$, the respective couplings must be scaled as $g_{d_{1}}=g_{d_{2}} \sqrt{d_{1} / d_{2}}$ in order to compare a calculation with $d=d_{1}$ to one with $d=d_{2}$. The respective forces must be scaled on the one hand by $\left(d_{2} / d_{1}\right)^{2}$ due to their distance ${ }^{-2}$ dimension, but on the other hand, divided by $\left(d_{2} / d_{1}\right)$ to account for the intrinsic linear $N$-dependence discussed in Sect. E.1.3, so that overall $F_{d_{1}}=F_{d_{2}} d_{2} / d_{1}$. Once the couplings and forces are thus scaled to account for their non-zero dimensions, the calculations performed using different values of $a$ agree well; see the bottom right panel of Fig. 19. (This invariance with the lattice unit is valid independent of taking the $N \rightarrow 0$ limit.)
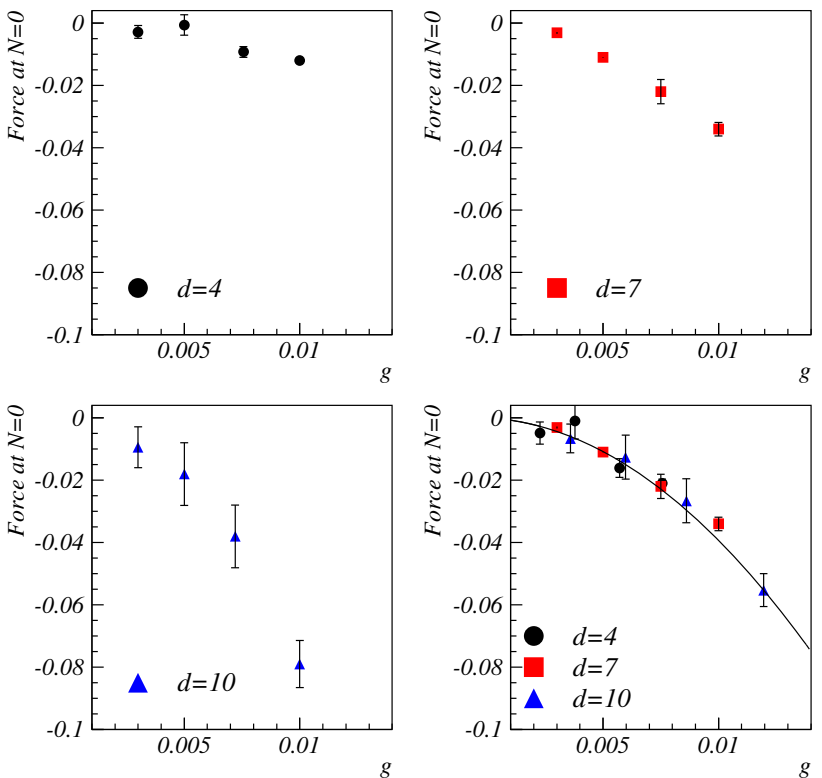

Fig. 19 Dependence of the simulation on lattice spacing. The Lagrangian of Eq. (12) is used. In the four panels, the $\mathrm{d}\left(G_{2 p}(x)-\right.$ $\left.G_{2 p}^{\text {free-field }}(x)\right) / \mathrm{d} x$ part of the force along $x$ is plotted as a function of the coupling $g$, in the classical $N \rightarrow 0$ limit. The number of lattice nodes between the two matter terms is $2 d=8$ (top left panel, circles), $2 d=14$ (top right panel, squares) and $2 d=20$ (bottom left panel, triangles). The bottom right plot shows these results for a same physical two-source system, after properly rescaling for the difference in physical lattice units. The three calculations using different physical lattice units agree well. The full curve is a second-order polynomial fit to the data

\section{F Recovering the post-Newtonian formalism}

We discussed in Sect. 5.3 how scalars fields could describe static systems. To verify that Eq. (12) provides an adequate static approximation of GR, we retrieve here the weakfield perturbative post-Newtonian formalism from the scalar Lagrangian in the static case of two bodies. We start from the Lagrangian of Eq. (12) truncated to first order in $\sqrt{16 \pi G}$ :

$$
\begin{aligned}
& \mathcal{L}=(\nabla \phi)^{2}+\sqrt{16 \pi G} \phi(\nabla \phi)^{2} \\
& -\sqrt{16 \pi G} \phi\left(M_{1} \delta^{(3)}\left(r-r_{1}\right)+M_{2} \delta^{(3)}\left(r-r_{2}\right)\right),
\end{aligned}
$$

where $r$ is the position of a test mass and $r_{1}$ and $r_{2}$ the positions of the two bodies of masses $M_{1}$ and $M_{2}$, respectively. The Euler-Lagrange equations yield

$$
\begin{aligned}
& \Delta \phi=-\frac{\sqrt{16 \pi G}}{2} \\
& \times \frac{M_{1} \delta^{(3)}\left(r-r_{1}\right)+M_{2} \delta^{(3)}\left(r-r_{2}\right)+(\nabla \phi)^{2}}{1+\sqrt{16 \pi G} \phi} .
\end{aligned}
$$

Assuming a weak field, $\sqrt{16 \pi G} \phi \ll 1$, and that the relative change of $\phi$ are small at long distances, i.e. $\nabla \phi \lesssim \phi$ (this is true for the zero order Newton solution and is justified a posteriori for the first order post-Newtonian solution), we 
can solve Eq. (20) to first order in $\sqrt{16 \pi G} \phi$ :

$$
\begin{aligned}
& \phi=-\frac{\sqrt{16 \pi G} M_{1}}{2\left(r-r_{1}\right)}-\frac{\sqrt{16 \pi G} M_{2}}{2\left(r-r_{2}\right)}+\frac{(16 \pi G)^{3 / 2}}{4} \\
& \times\left(\frac{M_{1}^{2}}{r-r_{1}}+\frac{M_{2}^{2}}{r-r_{2}}+2 \frac{M_{1} M_{2}}{\left(r-r_{1}\right)\left(r-r_{2}\right)}\right) .
\end{aligned}
$$

In the center of mass frame and suppressing the self-energy terms, we obtain the post-Newtonian potential energy in the static case:

$$
E=\frac{G M_{1} M_{2}}{r}\left(1-\frac{G\left(M_{1}+M_{2}\right)}{2 r}\right) .
$$

(To obtain Eq. 22, one must use the standard field normalization, $\phi \equiv\left(g_{\mu \nu}-\eta_{\mu \nu}\right)$, i.e. replacing $\phi$ by $\phi / \sqrt{16 \pi G}$. Many strong field effects are non-perturbative and cannot be obtained with such perturbative formalism. However, the exercise above validates the use of Eq. (12) for static nonperturbative calculations.

\section{References}

1. J. Greensite, An introduction to the confinement problem. In Lecture Notes in Physics, vol. 821 (Springer, Heidelberg, 2011)

2. C.D. Roberts, arXiv:1203.5341 [nucl-th]

3. S.J. Brodsky, G.F. de Tramond, H.G. Dosch, C. Lorce, Phys. Lett. B 759, 171 (2016)

4. R.B. Tully, J.R. Fisher, Astron. Astrophys. 54, 661 (1977)

5. S.S. McGaugh, J.M. Schombert, G.D. Bothun, W.J.G. de Blok, Astrophys. J. 533, L99 (2000)

6. A. Deur, Mon. Not. R. Astron. Soc. 438, 1535 (2014)

7. D. Clowe, M. Bradac, A.H. Gonzalez, M. Markevitch, S.W. Randall, C. Jones, D. Zaritsky, Astrophys. J. 648, L109 (2006)

8. M. Frasca, J. Nonlinear Math. Phys. 18, 291 (2011)

9. W. Buchmuller, A. Jakovac, Nucl. Phys. B 521, 219 (1998)

10. J. Zinn-Justin, arXiv:hep-ph/0005272

11. R.P. Feynman, Rev. Mod. Phys. 20, 367 (1948)
12. M. Kac, Trans. Am. Math. Soc. 65(1), 1 (1949)

13. K.G. Wilson, Phys. Rev. D 10, 2445 (1974)

14. L. Lehner, F. Pretorius, ARA\&A 52, 661 (2014)

15. P. Hoyer, arXiv:0909.3045 [hep-ph]

16. D.D. Dietrich, P. Hoyer, M. Jrvinen, Phys. Rev. D 87, 065021 (2013)

17. M. Frasca, Phys. Lett. B 670, 73 (2008)

18. M. Frasca, Mod. Phys. Lett. A 24, 2425 (2009)

19. A. Salam, IC/74/55 (1974)

20. A. Zee, in Quantum Field Theory in a Nutshell (Princeton University Press, Princeton, 2003)

21. M. Fierz, W. Pauli, Proc. R. Soc. A 173, 211 (1939)

22. A. Einstein, L. Infeld, B. Hoffmann, Ann. Math. 39, 65 (1938)

23. A. Deur, S.J. Brodsky, G.F. de Teramond, Prog. Part. Nucl. Phys. 90, 1 (2016)

24. S.J. Brodsky, G.F. de Teramond, H.G. Dosch, J. Erlich, Phys. Rep. 584, 1 (2015)

25. S.J. Brodsky, G.F. de Teramond, A. Deur, Phys. Rev. D 81, 096010 (2010)

26. A. Deur, S.J. Brodsky, G.F. de Teramond, Phys. Lett. B 750, 528 (2015)

27. A. Deur, S.J. Brodsky, G.F. de Teramond, Phys. Lett. B 757, 275 (2016)

28. A. Deur, V. Burkert, J.P. Chen, W. Korsch, Phys. Lett. B 650, 244 (2007)

29. A. Deur, V. Burkert, J.P. Chen, W. Korsch, Phys. Lett. B 665, 349 (2008)

30. E. Eichten, K. Gottfried, T. Kinoshita, J.B. Kogut, K.D. Lane, T.M. Yan, The spectrum of charmonium. Phys. Rev. Lett. 34, 369 (1975)

31. E. Eichten, K. Gottfried, T. Kinoshita, J.B. Kogut, K.D. Lane, T.M. Yan, Phys. Rev. Lett. 36, 1276 (1976)

32. E. Eichten, K. Gottfried, T. Kinoshita, K.D. Lane, T.M. Yan, Charmonium: the model. Phys. Rev. D 17, 3090 (1978)

33. E. Eichten, K. Gottfried, T. Kinoshita, K.D. Lane, T.M. Yan, Phys. Rev. D 21, 313 (1980)

34. E.J. Eichten, K. Lane, C. Quigg, B meson gateways to missing charmonium levels. Phys. Rev. Lett. 89, 162002 (2002)

35. G.S. Bali, QCD forces and heavy quark bound states. Phys. Rep. 343, 1 (2001)

36. M. Frasca, arXiv:1506.04612 [hep-ph]

37. A. Deur, Phys. Lett. B 676, 21 (2009)

38. T. Regge, Nuovo Cim. 14, 951 (1959) 